\title{
Improving effectiveness of spare parts supply by additive manufacturing as dual sourcing option
}

\author{
N. Knofius ${ }^{1}$ (D) - M. C. van der Heijden ${ }^{1}$ - A. Sleptchenko ${ }^{2}$ 'W. H. M. Zijm ${ }^{1}$
}

Received: 11 January 2019 / Accepted: 11 September 2020 / Published online: 9 October 2020

(C) The Author(s) 2020

\begin{abstract}
The low-volume spare parts business is often identified as a potential beneficiary of additive manufacturing (AM) technologies. Currently, high AM unit costs or low $\mathrm{AM}$ part reliabilities deem the application of AM economical inferior to conventional manufacturing $(\mathrm{CM})$ methods in most cases. In this paper, we investigate the potential to overcome these deficiencies by combining AM and CM methods. For that purpose, we develop an approach that is tailored toward the unique characteristics of dual sourcing with two production methods. Opposed to the traditional dual sourcing literature, we consider the different failure behavior of parts produced by AM and CM methods. Using numerical experiments and a case study in the aviation industry, we explore under which conditions dual sourcing with AM performs best. Single sourcing with AM methods typically leads to higher purchasing and maintenance costs while single sourcing with CM methods increases backorder and holding costs. Savings of more than $30 \%$ compared to the best single sourcing option are possible even if the reliability or unit costs of a part sourced with AM are three times worse than for a CM part. In conclusion, dual sourcing methods may play an important role to exploit the benefits of AM methods while avoiding its drawbacks in the low-volume spare parts business.
\end{abstract}

Keywords Inventory $\cdot$ Dual sourcing $\cdot$ Spare parts $\cdot$ Digital manufacturing $\cdot 3 \mathrm{D}$ printing

N. Knofius

n.knofius@utwente.nl

1 Industrial Engineering and Business Information Systems (IEBIS), Faculty of Behavioural Management and Social Sciences, University of Twente, P.O. Box 217, 7500 AE Enschede, The Netherlands

2 Khalifa University, Masdar Institute, Abu Dhabi, United Arab Emirates 


\section{Introduction}

Spare parts inventories are essential to keep downtime of advanced capital goods within reasonable limits, cf. Sherbrooke (2004), and Van Houtum and Kranenburg (2015). Investments in spare parts inventories can be huge, as the assortment contains many different items, among which are many expensive parts, slow movers, and long lead time items. This situation holds in particular for parts manufactured using conventional manufacturing (CM) technologies like milling, drilling, or injection molding.

Additive manufacturing (AM), also referred to as 3D printing, has increasingly matured to a point where it can be an alternative for spare parts production. The potential is exemplified by Airbus, an aircraft manufacturer, which reported that its A350 XWB aircraft contains more than 2700 printed parts (Airbus 2016). The unique process of AM in which raw materials are added layer upon layer to build complete parts at once is solely based on digital drawings. Therefore, AM leads to additional design freedom and possibly better part characteristics such as lower weight without sacrificing the structural strength. From a logistical point of view, it is interesting that part-specific tools or long setup times are atypical for AM methods. Especially in the low-volume spare parts, business lead times may be reduced substantially, and as a consequence decrease or even avoid the need for safety stocks.

However, it is doubtful whether AM methods will replace CM methods. Instead, it is more likely that AM methods will complement CM methods (Holweg 2015). Lead time reductions alone do not compensate for high AM unit costs or low and uncertain reliabilities. Unresolved issues such as unclear liability or non-standardized certification processes cause additional obstacles. While rapid regulatory advancements may overcome the latter obstacles soon, high unit costs and reliability concerns are more likely to prevail. Specifically, AM often suffers from the additional costs for post-processing and quality checks, high material costs, high equipment costs, and high process variability, cf. Frazier (2014) and Book and Sangid (2016).

In this paper, we study the value of sourcing spare parts with a mix of AM and CM methods. That is, depending on the situation one may decide whether to source a spare part with AM or CM methods. In the traditional dual sourcing literature, costs are higher and lead times are shorter for the fast supply option. In our case, this does not necessarily hold true, and more importantly: we take into account that AM parts may show a different failure behavior than CM parts. This extension seems inevitable given that not only the production process but also design and used material are typically different for both sourcing modes, cf. Wits et al. (2016). As a consequence, the sourcing decision will impact future demand, which makes the overall trade-off more complex. For example, fast resupply using AM helps reducing downtime in the short run, but in the long run a higher AM failure rate may offset this benefit. This aspect cannot be analyzed by any dual sourcing model available in the literature to the best of our knowledge, cf. Sect. 2.

As we will show, the flexibility of selecting between AM and CM methods holds the potential of significant cost savings-even if AM part characteristics such as 
piece price or reliability are inferior to the $\mathrm{CM}$ version. For example, if the AM item fails three times more often than the CM item on average, there are still realistic cases where dual sourcing offers cost savings of up to $30 \%$, cf. Sect. 4. Furthermore, we find that dual sourcing with AM is most promising for cases that are often observed in the spare parts business for capital goods: low demand rates, high holding and downtime costs. A case study conducted at an OEM in the aerospace industry broadens our results and exemplifies how other practical considerations such as design costs and operational cost savings may influence the value of dual sourcing with AM. In particular, we exemplify that the AM benefit of short lead times appears most interesting for parts that are less interesting for design improvement with AM.

The remainder of the paper is organized as follows. In Sect. 2, we position our work within the literature and clarify our contribution. Afterward, in Sect. 3, we develop a specific dual sourcing model and explain its evaluation and optimization. In Sect. 4, we conduct numerical experiments to study the value of dual sourcing in the spare parts business. Section 5 demonstrates the application of our model in practice. We close with Sect. 6, in which we suggest directions for future research.

\section{Literature review}

We discuss two literature streams. In Sect. 2.1, we review the dual sourcing literature where we focus on selected papers and refer to Minner (2003) and Zhou and Yang (2016) for a more extensive discussion. In Sect. 2.2, we position our paper in the fleet maintenance literature and recommend related reviews by Wang (2002) and Nicolai and Dekker (2008). We close with Sect. 2.3 in which we discuss models that quantify the value of AM spare parts supply.

\subsection{Dual sourcing}

Dual sourcing models typically distinguish between two supply options: one that is inexpensive but with a long resupply lead time (regular supply), and one that is expensive but with a short resupply lead time (expedited order). The first contribution to the dual sourcing literature was made by Barankin (1961), who discusses a single-period model with emergency shipments. Whittemore and Saunders (1977) consider the periodic review case with deterministic lead times. They show that as soon as lead times between both supply options differ by more than one period, the optimal policy depends on delivery time and quantity of the in-transit parts. Although dynamic programming methods allow solving such problems, the large state space leads to computational intractability even for medium-sized problems. Hence, more recent contributions are devoted to approximations of the optimal policy.

For the periodic review case, Veeraraghavan and Scheller-Wolf (2008) study a capacitated inventory model with deterministic lead times. They propose a dualindex policy that keeps track of one inventory position for each sourcing option. 
While the evaluation is partially based on simulation, the dual-index policy provides close to optimal results. Arts et al. (2011) extend the model and allow for stochastic lead times for the regular supply source. Also, their approach does not rely on simulation and thus decreases the computation time significantly. Scheller-Wolf et al. (2006) use a single-index policy where they monitor a single inventory position for each sourcing option. In case the inventory position is below the expedited target level, an order is placed to raise the inventory position to this level. Next, a regular order is used to bring the inventory position up to the regular target level. Their results show that the single-index policy performs comparably well but can be computed 25-60 times faster than the dual-index policy by Veeraraghavan and Scheller-Wolf (2008). Interestingly, Kiesmüller (2003), who proposed a dual-index policy for a remanufacturing system, argued that a singleindex policy is expected to perform significantly worse under some conditions. Thus, further investigation of the single-index policy proposed by Scheller-Wolf et al. (2006) may be worthwhile to clarify under which conditions a single-index policy performance is sufficiently well.

For the continuous review case, Moinzadeh and Schmidt (1991) propose a dual-index policy with deterministic lead times to determine the order quantity for both supply options. Using information about arrival times of outstanding orders, they keep orders from the expensive sourcing option limited. Song and Zipkin (2009) extend the model of Moinzadeh and Schmidt (1991) for multiple supply options and stochastic lead times. Therefore, they construct a queueing network with overflow bypass. Zhou and Yang (2016) introduce a single-index $(\mathrm{R}, \mathrm{nQ})$ policy and study a more general setting regarding fixed order costs, batch ordering, and compound Poisson demand. Axsäter (2007) proposes a decision rule for placing expedited orders. Therefore, the regular source is operated according to a single sourcing $(\mathrm{R}, \mathrm{Q})$ policy while, if the inventory level drops below zero, expedited orders are placed according to the decision rule. In followup papers, the decision rule is improved (Axsäter 2014) and the model is extended to incorporate the option of committed service times (Huang et al. 2011). Allon and Van Mieghem (2010) propose a tailored base-surge (TBS) policy with stochastic lead times. Under the TBS policy, goods are ordered at a constant rate from the cheap supply source while orders for the expensive supply source are issued according to a base stock policy. Song et al. (2017) propose an approach that finds the optimal policy for a continuous review system for special cases. Therefore, they transform the original problem into a simplified queueing system that shares the same optimal policy under specific conditions on the net inventory. For cases in which these conditions are violated, they exploit the results of the queueing system to construct a policy for the original system. Numerically they show that the resulting heuristic policy is close to optimal and typically outperforms the discussed methods of Song and Zipkin (2009) and Allon and Van Mieghem (2010).

To the best of our knowledge, none of many papers on dual sourcing addresses the impact of sourcing decisions on future demand which is essential for our analysis, cf. Sect. 1. Furthermore, for dual sourcing problems with supply modedependent failure behavior, the common technique to aggregate state information 
to approximate ordering policies becomes more challenging. For instance, various heuristics base order decisions on the inventory position (stock level plus intransit items) rather than the delivery time and size of each order. In our case, however, we have to distinguish between CM and AM items that are installed, in stock, and in resupply. Otherwise, it would not be possible to account for the impact of the order decision on future demand.

\subsection{Fleet maintenance}

A fleet describes a system of similar assets that share common technical features and work under the same operating conditions (Petchrompo and Parlikad 2019). Examples comprise aircraft, production systems, busses, or power transformers. A key modeling challenge constitutes an accurate representation of the asset dependencies. In the literature, these dependencies are categorized in economic, structural, stochastic, and resource dependencies Olde Keizer et al. (2017a). Most relevant for our paper is the resource dependency in which common resources, such as spare parts or tooling, are shared across a finite fleet of assets.

Park (1981) made an early contribution in this respect. He developed an optimization model in which spare parts demand depends on the number of operating systems. Later, spare parts management decisions were also considered in combination with maintenance decision. For example, Godoy et al. (2014) jointly optimize the preventive maintenance interval and the spare parts stock level. Another example is the paper by Olde Keizer et al. (2017b). They use a Markov decision process to analyze spare parts ordering and maintenance decisions based on the condition of a multi-component system. For more examples, we refer the reader to Van Horenbeek et al. (2013), and de Jonge and Scarf (2019).

Despite the large variety of contributions in the fleet maintenance domain, we are not aware of a paper in which multiple technical options are considered to replace a failed part in a fleet. In addition, we note that the related fleet maintenance literature is mostly concerned with operational planning decisions, whereas we primarily study a tactical planning decision, i.e., under which conditions is it worthwhile to use AM as a dual sourcing option.

\subsection{Quantitative models on the application of AM}

Here, we review quantitative results on the application of AM technology in the spare parts business and refer to Walter et al. (2004) and Holmström et al. (2010) for a conceptual discussion. Most quantitative contributions study the impact of sourcing spare parts with AM instead of CM methods. Liu et al. (2014) and Khajavi et al. (2014) investigate under which conditions a centralized or a decentralized deployment of AM equipment is preferable. They find that AM equipment costs, automation, and demand pattern influence the AM supply chain layout most. Liu et al. (2014) demonstrate that using AM instead of CM methods may support significant safety stock reductions. Furthermore, Li et al. (2017) show that carbon emissions are 
reducible with an AM supply chain layout. We focus on the case where AM parts are sourced from an external supplier. Expensive AM equipment and its required support infrastructure, see, e.g., Eyers and Potter (2017), render in-house production a less likely scenario for most service organizations.

Sirichakwal and Conner (2016) evaluate how using AM methods may influence the stock-out probability. Therefore, they assume that AM methods allow holding costs and lead time reductions. They find that holding cost reductions typically reduce the stock-out probability. In case of lead time reductions, the integrality of stock levels occasionally causes an increase in stock-out probability but overall has a positive effect as well. Given that they do not consider cost, the monetary effect of using AM for spare parts supply remains unclear.

Westerweel et al. (2018) study which AM part reliability and AM production costs level have to be achieved to reach a break-even point compared to a CM part. Overall, it turns out that a low AM part reliability is more restrictive than high AM production costs. Yet, they find that in the absence of AM performance benefits utilizing AM for spare parts supply alone often does not pay off. We take a different angle and study the value of combining CM with AM for spare parts supply.

Knofius et al. (2018) study the effects of redesigning spare parts with AM technology. Therefore, they consider the option to replace a set of components with fewer but therefore more complex AM parts. The changing spare part configuration next to reliability, lead time, and unit costs changes significantly influences service cost. Hence, they argue that a total cost perspective is required when judging about the effects of AM on spare parts supply. In this paper, we assume that the spare parts configuration remains unchanged but consider changes in reliability, unit costs, and lead times.

The paper closest related to our work is the one by Song and Zhang (2016), who consider the use of AM methods as an emergency channel that may produce spare parts on demand. Opposing to our model, stocking AM items is not allowed and thus causes a waiting time for each incoming demand. In their model, AM equipment is capacitated (modeled as an M/D/1 queue) but typically allows faster, though more expensive resupply than the CM source. Later, they find that AM equipment utilization typically remains low and therefore may support our assumption of an uncapacitated printing source. Also, they assume that AM parts have the same failure behavior as CM parts which is a fundamental difference compared to our model. Overall, they find that the production of parts on demand with AM methods leads to cost savings and inventory reductions compared to the application of CM methods only; especially, for situations with large part variety, the savings potential was significant.

The contribution of the paper is as follows:

1. We study a new dual sourcing problem, in which future demand depends upon the sourcing options being used via different failure rates for different spare part types. We explain why this type of dual sourcing problem becomes relevant with the emergence of AM technology.

2. In numerical experiments and a case study in the aviation industry, we explore under which conditions AM holds value as dual sourcing option. 
3. We expose that printing on demand is typically not practical for downtime critical spare parts. Instead, spare parts stocks remain essential to realize high system availability.

4. We show that the current focus on design improvements with AM may cause an undervaluation of the logistical benefits with AM. That is, scenarios most interesting for design improvements appear less promising for improving the effectiveness of spare parts supply with AM.

\section{Model}

We consider a company that is responsible for maintaining an installed base of a particular system type with a single stock point. Here, we focus on one specific component which can be sourced from either a CM or an AM supplier. Both options are characterized by differences in part reliability, unit costs, and replenishment lead time. The company is uncertain on whether and how to combine both sourcing options. In particular, it is unclear whether to replace a failed part with a CM or AM spare part (if both spare parts versions are in stock) and when to reorder from the $\mathrm{CM}$ or the AM supplier.

Certainly, if the stock is (nearly) depleted, it may appear better to order the AM version to exploit the typically faster AM replenishment lead time. In other cases, though, sufficient stock might be available. Here, despite a long lead time, it may be better to order a usually more reliable and cheaper CM version of the item instead. These trade-offs are further complicated by the difference in part reliability: if increasingly more AM parts are operated, it is likely that future demand increases (assuming that AM items fail more often).

We are investigating under which conditions it is advisable to use AM as dual sourcing option. Therefore, we develop a model which minimizes the long-run average cost by determining the optimal sourcing, maintenance, and inventory policy. In particular, we consider purchasing costs, maintenance costs, holding costs, and backorder costs. In principle, we can solve this problem by different methods. Probably, the most common approach is the utilization of a Markov Decision Process (MDP). Here, we employ a continuous-time Markov Chain analysis in combination with linear programming methods as we motivate in Sect. 3.4.

\subsection{Key assumptions}

Before outlining the model in detail, we describe and motivate the key assumptions.

\section{Failures follow a Poisson process}

This assumption appears justified given that we typically deal with mechanical parts that are designed to outlast the lifetime of the capital good or the preventive maintenance interval. For the latter case, preventive maintenance activities are typically scheduled well in advance and dedicated spare parts are usually 
ordered on demand. Hence, we only consider spare parts demand for corrective maintenance activities where failures are random in nature and may, for instance, be caused by unintended stress levels, imprudence during system usage, maintenance, shipping activities, or unobservable quality issues during the production. Nevertheless, the model can easily be extended to a wear-out process, for example, by modeling the failure behavior by an Erlang-2 distribution.

2. Base stock policy independent of the installed base composition.

In the dual sourcing literature, we often find replenishment policies other than one-for-one replenishment, cf. Sect. 2.1. In particular, many authors propose batch ordering which is motivated by high fixed order costs. For expensive, slowmoving items fixed order costs are typically insignificant, see, e.g., Van Houtum and Kranenburg (2015) and Hu et al. (2018); hence, a base stock policy seems most reasonable. In case of AM, the absence of tooling and setup costs further reduces the relevance of fixed costs. An additional complexity arises through the different failure behavior of CM and AM items. This characteristic might cause a dynamic stocking policy to be optimal, i.e., the optimal stock level depends on the installed base composition. However, as we argue in Sect. 3.6, this effect is less relevant for slow-moving spare parts and thus is not considered in this paper. Yet, we show how our model could be extended to analyze this aspect in 'Appendix 4.'

3. Resupply rates are exponentially distributed.

We justify this assumption with two key reasons: first, this assumption facilitates the use of a continuous-time Markov chain analysis. A drawback of exponential resupply lead times may be that ordering many items at once reduces the mean waiting time for parts (= lead time/number of parts ordered). Obviously, that is not realistic. However, we do not allow for ordering multiple parts at once, since we apply one-for-one replenishment policies only, cf. Assumption 2. Second, it is known that the performance of inventory systems for slow-moving spare parts is not sensitive to the shape of the lead time distribution, cf. Alfredsson and Verrijdt (1999), Alvarez et al. (2013) and Sleptchenko and Van der Heijden (2016). As we show using a simulation study in 'Appendix 1,' the same holds true for our specific problem.

4. Uncapacitated AM equipment.

As justified in Sect. 3.2, we assume that spare parts are sourced from an external supplier whose capacity is shared between multiple customers. A customer usually has limited information about congestion but instead observes the gross throughput time (including possible waiting times). Hence, we also regard the gross throughput time of the AM process as input to our model rather than the net manufacturing times. The same approach is typically applied in METRIC models for spare part optimization that are based on M/G/ $\infty$ queueing models for repair shops, cf. Sherbrooke (2004). Furthermore, as shown by Song and Zhang (2016), even in case AM production is conducted on demand, the capacity utilization remains low (less than $8 \%$ on average and $25 \%$ in the most extreme scenarios). Hence, the likelihood of congestion at the AM source is low. Finally, the growing availability of general-purpose AM capacity gives customers additional flexibility to avoid long or uncertain lead times. 


\subsection{Notation and model outline}

We model a single-item inventory system which serves an installed base of $k$ systems, where each system requires one unit of the item to be operational. The CM and AM version fails according to a Poisson process with rates $\lambda_{C}$ and $\lambda_{A}$, respectively. Upon failure, we encounter maintenance costs $m$ which include aspects such as spare parts transportation costs, repair costs, and service engineer costs. Maintenance costs are independent of the failed and replacement part. The unit costs of a new CM or AM part are denoted by $c_{C}$ and $c_{A}$, and the resupply rates are exponentially distributed with rates $\mu_{C}$ and $\mu_{A}$.

The installed base is supported by a single stockpoint carrying $S$ non-repairable spare parts. Upon failure, a CM or AM spare part is taken from stock, and a new $\mathrm{CM}$ or AM part is ordered immediately. Hence, the total number of parts in the system (operational, in stock or in resupply) equals $N=k+S$. In case we run out of stock, demand is backlogged, and we incur backorder costs $b$ per item per time unit. In fact, these backorder costs can be interpreted as penalty costs for system downtime. Otherwise, if a spare part is available, the replacement of the failed part takes place instantaneously (maintenance related downtime is covered in the maintenance costs). Holding costs are modeled as a fraction $\kappa$ of the associated unit costs of the items in stock.

The tuple $\left(n_{C, \mathbf{i}}, n_{A, \mathbf{i}}, r_{C, \mathbf{i}}, r_{A, \mathbf{i}}, s_{C, \mathbf{i}}, s_{A, \mathbf{i}}\right)$ describes the state $\mathbf{i}$ of the inventory system where $n_{C, \mathbf{i}}\left(n_{A, \mathbf{i}}\right)$ refers to the number of CM (AM) parts in operation, $r_{C, \mathbf{i}}\left(r_{A, \mathbf{i}}\right)$ refers to the number of CM (AM) parts in resupply, and $s_{C, \mathbf{i}}\left(s_{A, \mathbf{i}}\right)$ refers to the number of $\mathrm{CM}$ (AM) spare parts in stock. The set of feasible states is equal to

$$
\begin{aligned}
\Omega=\{ & \left(n_{C, \mathbf{i}}, n_{A, \mathbf{i}}, r_{C, \mathbf{i}}, r_{A, \mathbf{i}}, s_{C, \mathbf{i}}, s_{A, \mathbf{i}}\right): \\
& n_{C, \mathbf{i}}+n_{A, \mathbf{i}}+r_{C, \mathbf{i}}+r_{A, \mathbf{i}}+s_{C, \mathbf{i}}+s_{A, \mathbf{i}}=N \\
& s_{C, \mathbf{i}}+s_{A, \mathbf{i}}=\max \left\{N-k-r_{C, \mathbf{i}}-r_{A, \mathbf{i}} ; 0\right\} \\
& n_{C, \mathbf{i}}+n_{A, \mathbf{i}} \leq k \\
& \left.n_{C, \mathbf{i}}, n_{A, \mathbf{i}}, r_{C, \mathbf{i}}, r_{A, \mathbf{i}}, s_{C, \mathbf{i}}, s_{A, \mathbf{i}} \geq 0\right\}
\end{aligned}
$$

Note that the definition of the state space excludes degenerated transitions. So, in case $n_{C, \mathbf{i}}+n_{A, \mathbf{i}}<k$ it is impossible to have items in stock and a failed item always has to be replaced immediately. Overall, the state space grows polynomially with the installed base size $k$ and maximum number of spare parts $S$. More precisely, the state space size is equal to:

$$
\left(\begin{array}{c}
S+4-1 \\
4-1
\end{array}\right)(k+1)+\sum_{a=1}^{k} a(k+S+2-a)
$$

where the first term subsumes states with no backlog and the summation states with backlog.

Upon failure of an item, one has to take two decisions. First, whether to use an $\mathrm{AM}$ or a $\mathrm{CM}$ item from stock (if possible) to replace the failed item (maintenance decision). Note that the item which failed does not need to be replaced with the 
same item version. Second, we have to decide whether to reorder an AM or a CM item to replenish the stock (sourcing decision). Optimal decisions are dependent on the state $\mathbf{i}$.

We define a matrix $\mathbf{X}$ to represent the decisions for all states, where each column corresponds to a certain state $\mathbf{i}$, and each row to a decision option $c$, defined as follows:

- $c=1$ : take AM version from stock (if possible), and order AM version.

- $c=2$ : take CM version from stock (if possible), and order AM version.

- $c=3$ : take AM version from stock (if possible), and order CM version.

- $c=4$ : take $\mathrm{CM}$ version from stock (if possible), and order CM version.

where each matrix element $x_{c}(\mathbf{i})$ represents a binary variable that is equal to 1 if we choose decision option $c$ in state $\mathbf{i}$ and 0 otherwise. Note that in cases where $s_{C, \mathbf{i}}+s_{A, \mathbf{i}}=0$ it is indifferent whether to choose $c=1$ or $c=2$. The same holds for the choice between $c=3$ and $c=4$. In Table 1, we summarize the notation.

Table 1 Notation overview

\begin{tabular}{|c|c|}
\hline Notation & Explanation \\
\hline$k$ & Installed base size \\
\hline$\lambda_{C}$ & $\mathrm{CM}$ failure rate \\
\hline$\lambda_{A}$ & AM failure rate \\
\hline$\mu_{C}$ & $\mathrm{CM}$ resupply rate \\
\hline$\mu_{A}$ & AM resupply rate \\
\hline$c_{C}$ & $\mathrm{CM}$ piece price \\
\hline$c_{A}$ & AM piece price \\
\hline$o_{A}$ & Operational cost savings per AM part per time unit \\
\hline$m$ & Maintenance costs \\
\hline$b$ & Backorder costs per time unit \\
\hline$d$ & Depreciation costs per time unit \\
\hline$\kappa$ & Holding costs as fraction of the piece price per time unit \\
\hline$S$ & Maximum number of spare parts circulating in the inventory system \\
\hline$N$ & Total number of parts $(N=k+S)$ \\
\hline $\mathbf{i} \in \Omega$ & Six-dimensional state vector, $\left(n_{C, \mathbf{i}}, n_{A, \mathbf{i}}, r_{C, \mathbf{i}}, r_{A, \mathbf{i}}, s_{C, \mathbf{i}}, s_{A, \mathbf{i}}\right)$ \\
\hline$n_{C, \mathbf{i}}$ & Number of CM parts in operation \\
\hline$n_{A, \mathbf{i}}$ & Number of AM parts in operation \\
\hline$r_{C, \mathbf{i}}$ & Number of CM parts in resupply \\
\hline$r_{A, \mathbf{i}}$ & Number of AM parts in resupply \\
\hline$s_{C, \mathbf{i}}$ & Number of CM parts in stock \\
\hline$s_{A, \mathbf{i}}$ & Number of AM parts in stock \\
\hline$c \in\{1,2,3,4\}$ & Maintenance and sourcing decision \\
\hline
\end{tabular}




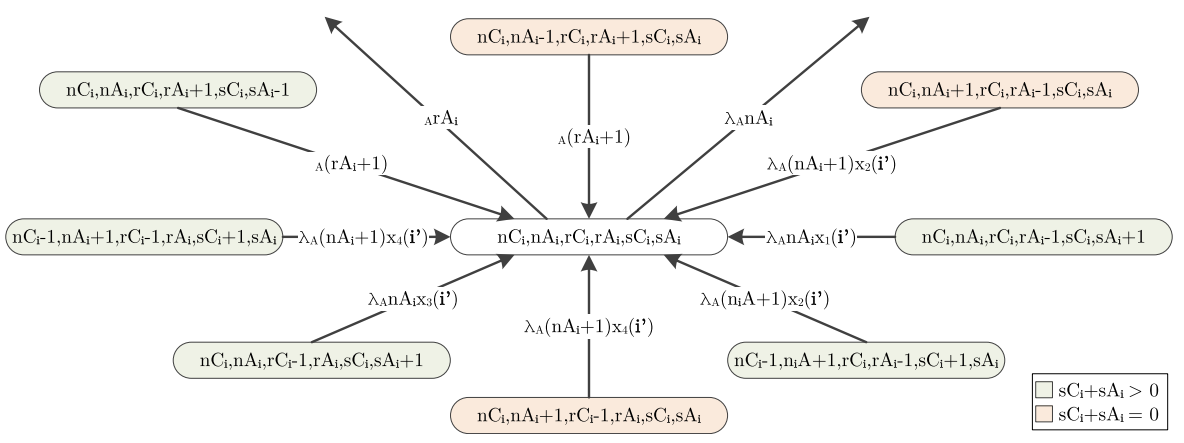

Fig. 1 Possible transitions to and from state $\left(n_{C, \mathbf{i}}, n_{A, \mathbf{i}}, r_{C, \mathbf{i}}, r_{A, \mathbf{i}}, s_{C, \mathbf{i}}, s_{A, \mathbf{i}}\right)$ if an AM item arrives or fails

\subsection{Evaluation}

Given $\mathbf{X}, k$ and $S$, the model is evaluated using a continuous-time Markov Chain. In Fig. 1, we show the ten possible transitions to and from state $\left(n_{C, \mathbf{i}}, n_{A, \mathbf{i}}, r_{C, \mathbf{i}}, r_{A, \mathbf{i}}, s_{C, \mathbf{i}}, s_{A, \mathbf{i}}\right)$ for the case where an AM part arrives or fails. The ten transitions associated with the arrival or failure of a CM part are omitted but exhibit the same pattern. We use $\mathbf{i}^{\prime}$ to refer to the associated predecessor states of $\left(n_{C, \mathbf{i}}, n_{A, \mathbf{i}}, r_{C, \mathbf{i}}, r_{A, \mathbf{i}}, s_{C, \mathbf{i}}, s_{A, \mathbf{i}}\right)$. Furthermore, if the maintenance decision is indifferent (i.e., no stock available), as we elaborated in the previous section, we use $c=2$ and $c=4$ as default in Fig. 1 .

Predecessor states in which the total stock is depleted are colored in red in Fig. 1. For these states, a transition causes an increase or decrease in the number of operating components, i.e., $n_{C, \mathbf{i}}+n_{A, \mathbf{i}}$ changes. Predecessor states in which stock is available are colored in green in Fig. 1. For these states, a transition does not affect the total number of operating components $\left(n_{C, \mathbf{i}}+n_{A, \mathbf{i}}\right)$ but only increases or decreases the stock, i.e., $s_{C, \mathbf{i}}+s_{A, \mathbf{i}}$ changes. However, as is the case for the predecessor state located in the bottom right corner, a transition might cause a change of the installed base composition. For every predecessor state, a transition causes a change in the resupply pipeline, i.e., $r_{C, \mathbf{i}}+r_{A, \mathbf{i}}$ changes.

The balance equations (cf. 'Appendix 1') directly follow from the transitions illustrated in Fig. 1 and, in combination with the normalization equation, allow the computation of the state probabilities $p_{\mathbf{i}}$ with common methods. The result is captured by the column vector $\mathbf{p}$ with elements $p_{\mathbf{i}}$.

After $\mathbf{p}$ was determined, the long-run average cost $C$ is computable by $\mathbf{g}^{\mathbf{T}} \mathbf{p}$ with $\mathbf{g}$ representing an $|\Omega|$-dimensional column vector of cost $g_{\mathbf{i}}$ in state $\mathbf{i}$. Given that we consider purchasing, maintenance, holding, and backorder costs, the total expected cost, given the system is in state $\mathbf{i}$, is given by

$$
\begin{aligned}
g_{\mathbf{i}}= & \mu_{C} c_{C} r_{C, \mathbf{i}}+\mu_{A} c_{A} r_{A, \mathbf{i}}+m\left(\lambda_{C} n_{C, \mathbf{i}}+\lambda_{A} n_{A, \mathbf{i}}\right) \\
& +\kappa\left(c_{C} s_{C, \mathbf{i}}+c_{A} s_{A, \mathbf{i}}\right)+b\left(k-n_{C, \mathbf{i}}-n_{A, \mathbf{i}}\right)
\end{aligned}
$$


Later, in Sects. 3.7 and 3.8 we also explain how additional AM design cost and operational cost savings can be considered. Note that, we account for the additional AM design cost separately from the model since they are independent of the sourcing and maintenance decision. Furthermore, as we explain in Sect. 3.8, we exclude operational cost savings from the default model because they are highly case dependent and thus unsuitable for a generic assessment. However, during the case study in Sect. 5, operational cost savings are taken into account as well.

\subsection{Optimization of the sourcing and maintenance policy}

Formally, the optimization problem can be expressed as Problem 1:

$$
\begin{array}{ll}
\underset{\mathbf{X}}{\operatorname{minimize}} & \mathbf{g}^{\mathbf{T}} \mathbf{p} \\
\text { subject to } & \mathbf{Q}(\mathbf{X}) \mathbf{p}=\mathbf{0} \\
& \mathbf{1}_{|\Omega|}^{\mathbf{T}} \mathbf{p}=1 \\
& \mathbf{p} \geq \mathbf{0} \\
& \mathbf{1}_{\mathbf{4}}^{\mathbf{T}} \mathbf{X}=\mathbf{1}_{|\Omega|}^{\mathbf{T}} \\
& x_{c}(\mathbf{i}) \in\{0,1\} \quad \forall c, \mathbf{i}
\end{array}
$$

where $\mathbf{Q}(\mathbf{X}) \mathbf{p}=\mathbf{0}$ represents the balance equations with matrix $\mathbf{Q}(\mathbf{X})$ describing the generator of the Markov process and $\mathbf{0}$ denoting an $|\Omega|$-dimensional vector of zeros. Furthermore, $\mathbf{1}_{|\Omega|}^{\mathbf{T}} \mathbf{p}=1$ defines the normalization equation with $\mathbf{1}_{\mathbf{z}}$ being an $z$-dimensional column vector of ones (here $z=|\Omega|$ or $z=4$ ).

Given the product $x_{c}(\mathbf{i}) p_{\mathbf{i}}$ in the balance equations (cf. Appendix 1), Problem 1 is nonlinear and therefore less computationally tractable. Therefore, we transform (1) into an equivalent linear formulation. The key step of the transformation is the rearrangement of the balance equations and the substitution of the product $x_{c}(\mathbf{i}) p_{\mathbf{i}}$ with the variable $y_{c}(\mathbf{i})$. One may interpret $y_{c}(\mathbf{i})$ as the long-run fraction of the time that the system is in state $\mathbf{i}$ and action $c$ is chosen. This operation allows us to redefine Problem 1 as follows:

$$
\begin{array}{ll}
\underset{\mathbf{Y}}{\operatorname{minimize}} & \mathbf{g}^{\mathbf{T}} \mathbf{p} \\
\text { subject to } & \sum_{c=1}^{4} \mathbf{L}_{\mathbf{c}} \mathbf{y}_{\mathbf{c}}+\mathbf{M p}=\mathbf{0} \\
& \mathbf{1}_{|\Omega|}^{\mathbf{T}} \mathbf{p}=1 \\
& \mathbf{p} \geq \mathbf{0} \\
& \mathbf{1}_{\mathbf{4}}^{\mathbf{T}} \mathbf{Y}=\mathbf{p}^{\mathbf{T}} \\
& y_{c}(\mathbf{i}) \geq 0 \quad \forall c, \mathbf{i}
\end{array}
$$

where matrix $\mathbf{L}_{\mathbf{c}}$ contains all transition rates dependent on decision option $c$, and matrix $\mathbf{M}$ contains all transition rates independent of decision option $c$. For example, as shown in 'Appendix 2,' $\mathbf{M}$ contains the last four terms in the balance equations. Furthermore, we use $\mathbf{y}_{\mathbf{c}}$ to describe an $|\Omega|$-dimensional column vector with elements $y_{c}(\mathbf{i})$ and $\mathbf{Y}$ to describe a matrix with row vectors $\mathbf{y}_{\mathbf{c}}$. 
Fig. 2 Examples of changing cost terms when $S$ increases

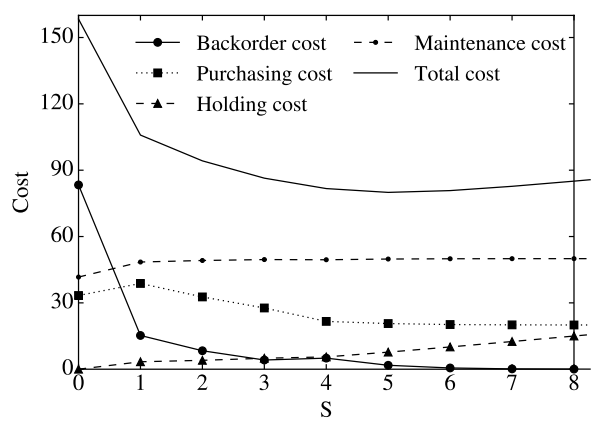

Fig. 3 Examples of failure type changes when $S$ increases

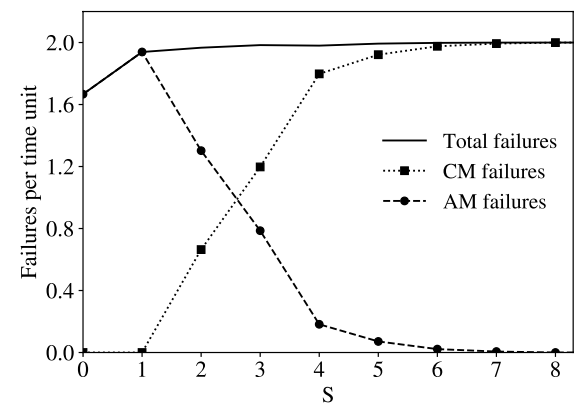

As a consequence, Problem (2) allows us to find the optimal values of $\mathbf{Y}$ and $\mathbf{p}$ with linear programming methods. Next, the optimal decisions $\mathbf{X}^{*}$ are recovered with the relation $x_{c}(\mathbf{i})=y_{c}(\mathbf{i}) / p_{\mathbf{i}}$. Note that in case $0<y_{c}(\mathbf{i})<p_{\mathbf{i}}$ we would obtain a randomized policy which violates the constraint $x_{c}(\mathbf{i}) \in\{0,1\} \forall c$, i of Problem 1 . Sleptchenko and Johnson (2015) however, show that, given a linear cost function, $x_{c}(\mathbf{i}) \in\{0,1\} \forall c$, i holds, i.e., we always obtain a deterministic policy with Problem (2).

Compared to an MDP approach, the described optimization procedure allows a more efficient model representation: instead of four equations per state (one for each action), we can represent the problem with two equations per state (balance equation and policy constraint). Furthermore, we do not need to discretize time, which reduces the number of possible transitions in each state.

\subsection{Optimization of the stocking policy}

The optimal base stock levels in the $(S-1, S)$ spare parts supply systems are usually determined with a greedy approach using convexity properties of the cost function in $S$, cf. Van Houtum and Kranenburg (2015). However, in the dual sourcing case presented here, a changing sourcing strategy with increasing $S$ causes a non-convex behavior of the individual costs terms. We exemplify this behavior for one instance (Parameter values: $d=250, c_{C}=10, c_{A}=20, \kappa=0.25, k=2, \lambda_{C}=1, \lambda_{A}=1$, $\left.\mu_{C}=1, \mu_{A}=5, m=25\right)$. 
Figure 2 shows different cost terms, and Fig. 3 shows the corresponding failure types. Observe that, with increasing $S$ relatively more $\mathrm{CM}$ items fail which is caused by the increasing preference to install CM rather than AM items. The switch from AM to CM produced components (with the increase in $S$ ), leading to the nonconvexity of the purchasing, backorder, and holding costs terms in the objective function.

At the same time, we were not able to find any example of non-convexity of the objective function. The fluctuations in the costs terms appear to compensate each other, and the total cost function remains convex in $S$. Therefore, we propose the following conjecture that will be used in the optimization procedure described below.

Conjecture 1 The optimal total cost $C(S)$ with fixed base stock level $S$ and optimized ordering policy $\mathbf{X}$ has only one local minimum for all $S \geq 0$, which consequently will be the global minimum.

To find the optimal number $S^{*}$ of spare parts circulating in the inventory system, we use a greedy approach to determine the optimal long-run average cost $C^{*}(0)$ given that $S=0$ first. Next, we set $S=1$ and determine $C^{*}(1)$. In case $C^{*}(0)<C^{*}(1)$, $S=0$ is the optimal base stock level. Otherwise, we continue to increment $S$ until $C^{*}(S)<C^{*}(S+1)$.

\subsection{Joint optimization}

It is also possible to jointly optimize the stocking policy with the sourcing and maintenance policy (cf. Appendix 2). In the joint optimization approach, we set an upper bound on the number of spare parts required and include the option not to order any part upon failure in state $\mathbf{i}$.

However, numerical experiments show that the joint optimization is computationally inferior to the greedy approach. The key reason for this characteristic is that it is difficult to find a tight upper bound on the number of spare parts, in combination with the fact that the computation times grow rapidly with the size of the state space. On the other hand, an advantage of the joint optimization approach is that this model can be extended to a dynamic stocking policy (cf. Appendix 3). Given different failure rates of the AM and CM version, it is likely that the optimal inventory level depends on the mixture of AM parts and CM parts in the installed base. So, the inventory level will be state dependent. The impact of such a dynamic spare parts inventory policy is a topic for further research. Yet, we suspect that the impact for slow-moving spare parts will be moderate since the optimal inventory level will not change significantly depending on the installed base.

\subsection{Design costs}

Designing two instead of one part version causes additional design costs but holds the promise of a more flexible inventory system. The trade-off between more desirable system parameters and additional costs has not received much attention in the 
literature but is regarded as a promising direction for future research, $\mathrm{cf}$. Hu et al. (2018). Often, it is assumed that the design costs are an increasing convex function of the part reliability, see Mettas (2000) and Öner et al. (2010). It remains unclear how other aspects such as AM resupply lead time and AM unit costs may also impact the design costs for the situation discussed in this paper. More research is needed to explore this relationship.

In order to include design costs in our analysis, we assume that design costs are depreciated over the remaining life cycle length. Depending on the applied depreciation method, the per time unit expense $d$ is then derived from the remaining life cycle length and design costs difference between single and dual sourcing.

\subsection{Operational cost savings}

Operational cost savings depend on the installed base composition. Thus, if we assume operational cost savings of $o_{A}$ per time unit and installed AM item, we can easily include the savings by adding the term $-o_{A} n_{A, \mathbf{i}}$ to the cost function $g_{\mathbf{i}}$. We will include operational cost savings in the case study presented in Sect. 5, but do not consider this cost factor in the numerical experiments. Operational cost savings are highly case dependent and thus appear unsuitable for a generic assessment of the effects of dual sourcing with AM. Furthermore, business cases highly dominated by AM-specific operational cost savings appear to diminish the value of dual sourcing as we demonstrate in Sect. 5 .

\section{Numerical experiments}

To gain insights on the value of dual sourcing with two different production methods, we carry out numerical experiments. In Sect. 4.1, we elaborate on the experimental settings and justify the selected parameter ranges. In Sect. 4.2, we present the results while Sect. 4.3 summarizes the key findings.

\subsection{Experimental settings}

The value of AM technology in the spare parts business is perceived highest for lowvolume applications, i.e., for applications where economies of scale are of minor importance (Khajavi et al. 2014; Liu et al. 2014). So, we focus on scenarios where the combination of failure rate and installed base size causes 1 to 7 demands with CM sourcing per year.

For expensive spare parts with infrequent demand, the replenishment lead time is often long. For example, Basten (2010) observes spare parts replenishment lead times of more than a year in the defense sector. In comparison, AM lead times are typically short which is exemplified by Liu et al. (2014) who report on data from the aerospace industry. For CM items, they find lead times between 1.5 and 8 months, whereas, for AM parts, they typically assume lead times of less than a month. AM lead times in the order of magnitude smaller than a week appear unrealistic - at least 
for metal printing processes. Pre- and post-processing activities seem inevitable for industrial applications today. For instance, usually a preheating and lengthy cooldown phase is necessary to ensure an acceptable part quality (Shouche et al. 2016). Furthermore, most companies rely on external AM suppliers which causes additional transportation times. Accordingly, we study AM lead times of 1 to 4 weeks and CM lead times of 2 to 6.5 month.

The AM piece price is typically higher than for CM parts. Not only does this situation relate to the novelty of industrial AM process, but also to the high raw material costs. Additionally, AM processes remain labor and skill intensive (Weller et al. 2015). As we already discussed in Sect. 1, the reliability of printed parts is often inferior compared to their CM counterparts. Thus, for various applications customized AM designs become necessary to achieve a comparable or even superior quality compared to the $\mathrm{CM}$ version. As we will also exemplify in the case study, one possible approach is the utilization of higher quality raw material. For other applications, a lower reliability might be acceptable also in view of an otherwise significantly higher AM piece price. For instance, in Sect. 4.2, we discuss AM applications at remote locations where printed, low-reliability parts are used as emergency solution to avoid high holding and downtime costs. Overall, we evaluate scenarios where the AM piece price varies between 1 and 3 times of the $\mathrm{CM}$ piece price.

We consider three different $\mathrm{CM}$ items in the numerical experiments. Item 1 exhibits a failure rate of $\lambda_{C}=0.02$ failures per month, i.e., a MTBF of about 4 years; a resupply rate of $\mu_{C}=0.5$ per month, i.e., an average lead time of two months, and a unit costs of $c_{C}=10$. Item 2 fails less frequently $\left(\lambda_{C}=0.015\right)$ and has a longer resupply lead time $\left(\mu_{C}=0.25\right)$. Item 3 exhibits an even lower failure rate $\left(\lambda_{C}=0.01\right)$ and longer resupply lead time $\left(\mu_{C}=0.15\right)$. All other parameters follow commonly observed conditions in the low-volume, high-variety spare parts business.

\subsection{Sensitivity analysis}

As we motivated in the previous section, we consider 8 parameters each with 3 different levels which leads to 6561 problem instances. Table 2 presents the results for the full-factorial design. Note that for each parameter value, we provide the average over the corresponding 2187 instances (6561/3). Furthermore, we provide the minimum and maximum values in Appendix 5. In the column 'Cost savings compared to' of Table 2, we show the percentage of cost which can be saved with a dual sourcing approach if compared to single sourcing with CM, single sourcing with AM, and the best instance specific single sourcing approach as average over associated instances. The column 'Best sourcing approach' shows the percentage of instances where single sourcing with CM, single sourcing with AM or dual sourcing, respectively, is the best approach. The column 'Base stock level' shows the average base stock level for different sourcing concepts over associated instances.

As an average over all instances, we obtain cost savings of more than $8 \%$ if we compare the dual sourcing cost with the cost of the best single sourcing option of each specific instance. In extreme cases, we even find instances with cost savings of 
Table 2 Numerical experiments as average over corresponding instances, where single sourcing with only CM is denoted by $C M$, single sourcing with only AM by $A M$, single sourcing with the best instance specific approach by Best, and dual sourcing by Dual

\begin{tabular}{|c|c|c|c|c|c|c|c|c|c|c|c|}
\hline \multirow{3}{*}{$\begin{array}{l}\text { Param- } \\
\text { eter }\end{array}$} & \multirow[t]{3}{*}{ Value } & \multirow[t]{3}{*}{ Unit } & \multirow{2}{*}{\multicolumn{3}{|c|}{$\begin{array}{l}\text { Cost savings } \\
\text { compared to }\end{array}$}} & \multirow{2}{*}{\multicolumn{3}{|c|}{$\frac{\text { Best sourcing }}{\text { approach }}$}} & \multicolumn{3}{|c|}{ Base stock } \\
\hline & & & & & & & & & \multicolumn{3}{|l|}{ level } \\
\hline & & & $\mathrm{CM}$ & $\mathrm{AM}$ & Best & $\mathrm{CM}$ & $\mathrm{AM}$ & Dual & $\mathrm{CM}$ & AM & Dual \\
\hline & 1 & - & $7 \%$ & $43 \%$ & $4 \%$ & $24 \%$ & $11 \%$ & $64 \%$ & 3.37 & 1.97 & 2.83 \\
\hline \multirow[t]{3}{*}{ Item } & 2 & - & $10 \%$ & $48 \%$ & $8 \%$ & $11 \%$ & $4 \%$ & $85 \%$ & 4.30 & 1.97 & 3.37 \\
\hline & 3 & - & $14 \%$ & $57 \%$ & $13 \%$ & $2 \%$ & $0 \%$ & $98 \%$ & 4.48 & 1.97 & 3.48 \\
\hline & 10 & $\begin{array}{c}\text { Installed } \\
\text { parts }\end{array}$ & $12 \%$ & $46 \%$ & $9 \%$ & $11 \%$ & $6 \%$ & $82 \%$ & 2.74 & 1.40 & 2.16 \\
\hline \multirow[t]{3}{*}{$k$} & 20 & $\begin{array}{c}\text { Installed } \\
\text { parts }\end{array}$ & $10 \%$ & $50 \%$ & $8 \%$ & $12 \%$ & $5 \%$ & $83 \%$ & 4.15 & 2.01 & 3.27 \\
\hline & 30 & $\begin{array}{c}\text { Installed } \\
\text { parts }\end{array}$ & $9 \%$ & $52 \%$ & $7 \%$ & $13 \%$ & $4 \%$ & $83 \%$ & 5.26 & 2.50 & 4.25 \\
\hline & 0.0175 & $\begin{array}{c}\text { Failures/ } \\
\text { month }\end{array}$ & $15 \%$ & $27 \%$ & $8 \%$ & $4 \%$ & $16 \%$ & $80 \%$ & 4.05 & 1.40 & 2.83 \\
\hline \multirow[t]{3}{*}{$\lambda_{A}$} & 0.035 & $\begin{array}{c}\text { Failures/ } \\
\text { month }\end{array}$ & $9 \%$ & $54 \%$ & $9 \%$ & $13 \%$ & $0 \%$ & $87 \%$ & 4.05 & 2.01 & 3.36 \\
\hline & 0.0525 & $\begin{array}{c}\text { Failures/ } \\
\text { month }\end{array}$ & $7 \%$ & $67 \%$ & $7 \%$ & $20 \%$ & $0 \%$ & $80 \%$ & 4.05 & 2.49 & 3.49 \\
\hline & 1 & $\begin{array}{l}\text { Supplies/ } \\
\text { month }\end{array}$ & $6 \%$ & $51 \%$ & $5 \%$ & $19 \%$ & $4 \%$ & $77 \%$ & 4.05 & 2.72 & 3.54 \\
\hline \multirow[t]{3}{*}{$\mu_{A}$} & 2 & $\begin{array}{l}\text { Supplies/ } \\
\text { month }\end{array}$ & $10 \%$ & $49 \%$ & $8 \%$ & $10 \%$ & $6 \%$ & $84 \%$ & 4.05 & 1.88 & 3.21 \\
\hline & 4 & $\begin{array}{l}\text { Supplies/ } \\
\text { month }\end{array}$ & $14 \%$ & $48 \%$ & $11 \%$ & $7 \%$ & $6 \%$ & $86 \%$ & 4.05 & 1.31 & 2.94 \\
\hline & 10 & Euro/part & $15 \%$ & $32 \%$ & $9 \%$ & $6 \%$ & $16 \%$ & $79 \%$ & 4.05 & 2.25 & 2.91 \\
\hline \multirow[t]{3}{*}{$c_{A}$} & 20 & Euro/part & $9 \%$ & $53 \%$ & $9 \%$ & $12 \%$ & $0 \%$ & $88 \%$ & 4.05 & 1.93 & 3.32 \\
\hline & 30 & Euro/part & $7 \%$ & $64 \%$ & $7 \%$ & $19 \%$ & $0 \%$ & $81 \%$ & 4.05 & 1.72 & 3.46 \\
\hline & 20 & $\begin{array}{l}\text { Euro/ } \\
\text { month }\end{array}$ & $4 \%$ & $50 \%$ & $3 \%$ & $34 \%$ & $5 \%$ & $61 \%$ & 2.63 & 0.90 & 2.31 \\
\hline \multirow[t]{3}{*}{$b$} & 200 & $\begin{array}{l}\text { Euro/ } \\
\text { month }\end{array}$ & $11 \%$ & $49 \%$ & $9 \%$ & $2 \%$ & $5 \%$ & $92 \%$ & 4.07 & 2.01 & 3.25 \\
\hline & 2000 & $\begin{array}{l}\text { Euro/ } \\
\text { month }\end{array}$ & $15 \%$ & $49 \%$ & $12 \%$ & $1 \%$ & $5 \%$ & $94 \%$ & 5.44 & 3.00 & 4.13 \\
\hline & 2 & $\begin{array}{l}\text { Euro/fail- } \\
\text { ure }\end{array}$ & $13 \%$ & $49 \%$ & $10 \%$ & $9 \%$ & $6 \%$ & $85 \%$ & 4.05 & 1.97 & 3.16 \\
\hline \multirow[t]{3}{*}{$m$} & 10 & $\begin{array}{l}\text { Euro/fail- } \\
\text { ure }\end{array}$ & $10 \%$ & $50 \%$ & $8 \%$ & $12 \%$ & $5 \%$ & $85 \%$ & 4.05 & 1.97 & 3.24 \\
\hline & 18 & $\begin{array}{l}\text { Euro/fail- } \\
\text { ure }\end{array}$ & $8 \%$ & $50 \%$ & $6 \%$ & $15 \%$ & $4 \%$ & $80 \%$ & 4.05 & 1.96 & 3.29 \\
\hline & 0.15 & - & $9 \%$ & $51 \%$ & $7 \%$ & $13 \%$ & $5 \%$ & $83 \%$ & 4.22 & 2.11 & 3.40 \\
\hline \multirow[t]{2}{*}{$\kappa$} & 0.2 & - & $10 \%$ & $49 \%$ & $8 \%$ & $13 \%$ & $5 \%$ & $82 \%$ & 4.04 & 1.95 & 3.22 \\
\hline & 0.25 & - & $11 \%$ & $48 \%$ & $9 \%$ & $11 \%$ & $6 \%$ & $83 \%$ & 3.89 & 1.85 & 3.07 \\
\hline
\end{tabular}


more than $30 \%$ which is remarkable because also for these cases the AM failure rate and the AM piece price are often two to three times higher than for the CM part.

Single sourcing with AM is significantly outperformed by dual sourcing, see the column 'Cost savings compared to $A M$ ' in Table 2. This result is explained by the following observations. First, an installed base composed of AM items typically causes a higher demand rate, which increases the maintenance and the purchasing costs, cf. Fig. 4. Second, given that we only order the usually more expensive AM item, the purchasing and the holding costs increase (the holding costs are a fixed fraction of the corresponding piece prices).

In this context, we also stress that printing spare parts on demand is no option for most instances. For example, even if the resupply lead time is equal to one week $\left(\mu_{A}=4\right)$, we find an average AM base stock level of 1.31 over associated instances, cf. Table 2. Hence, the common belief, see, e.g., Pérès and Noyes (2006), Campbell et al. (2012) and Thomas and Gilbert (2014), that the spare parts business may particularly benefit from a demand-driven production approach has to be reconsidered in this respect.

Single sourcing with $\mathrm{CM}$ is usually also outperformed by dual sourcing, see the column 'Cost savings compared to $C M$ ' in Table 2 which is caused by a reduction in the holding and the backorder costs with dual sourcing in comparison with single sourcing with $\mathrm{CM}$, cf. Fig. 4. Also, the purchasing and the maintenance costs increase with dual sourcing is marginal compared to single sourcing with CM. This finding relates to the flexibility to order from the AM source if the stock is (nearly) depleted while, otherwise, the typically cheaper and more reliable CM part is ordered. In fact, as shown in Fig. 5, the first order from the AM source usually only takes place if nearly no stock is left. Hence, in line with the dual sourcing literature, the AM source associated with a short resupply lead time usually functions as an emergency option.

Also because of this result, we less frequently observe the scenario that both item types are in stock. In total, only with a probability of about $4.6 \%$ we are in such a state. However, in case both item types are in stock, we typically use the CM item first (in about $92 \%$ of cases). This finding relates to the usually lower CM failure rate. Installing an item with a lower failure rate decreases the expected demand and thus leads to lower cost in the long-run. This result is further supported by the observation that a higher AM failure rate decreases the likelihood of installing an AM item first. Conversely, typically higher holding cost for the AM item does not sufficiently incentivize installing the AM item first.

The cost savings with dual sourcing are most sensitive to the AM resupply rate $\left(\mu_{A}\right)$ and the backorder costs $(b)$, if we study the column 'Cost savings compared to Best' in Table 2. This finding can be explained by the observation that both parameters primarily influence the holding and the backorder costs. As we just discussed, only by reducing either of these cost factors dual sourcing may outperform single sourcing with CM methods. In contrast, the cost savings potential with dual sourcing is less sensitive to the AM failure rate $\left(\lambda_{A}\right)$ and the AM piece price $\left(c_{A}\right)$, cf. column 'Cost savings compared to Best' in Table 2. So, dual sourcing appears to be a suitable vehicle to exploit the short AM resupply lead time. Negative effects of 
Fig. 4 Cost composition for different sourcing concepts as average over all instances

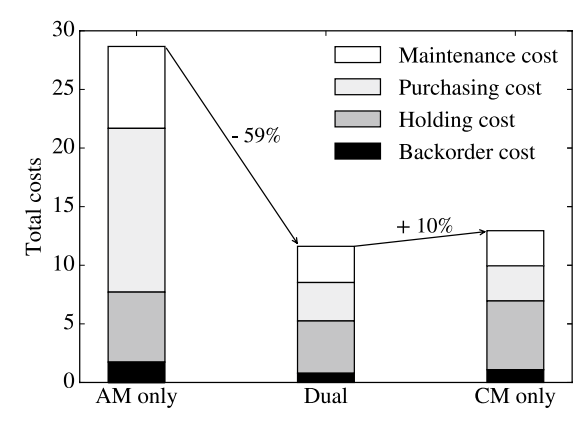

Fig. 5 Number of dual sourcing instances where we first order from the AM source if $\mathrm{x} \%$ of the total stock has been depleted

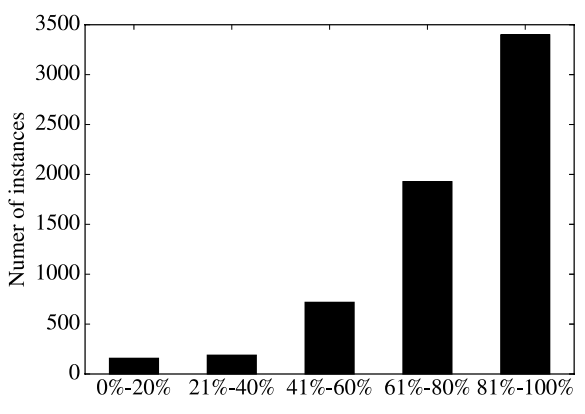

high AM unit costs and low AM part reliability turn out less crucial for a successful implementation.

As a consequence, various new service concepts are foreseeable. For instance, dual sourcing with AM may provide a good option to efficiently service downtime critical goods at remote locations. Today, long lead times and high inventory costs often cause high service cost. These may be reduced if locally printed-possibly less reliable_-spare parts become available as an emergency solution. First, applications can be found in defense organizations which experiment with mobile AM production facilities (McLearen 2015). A small sub-experiment may substantiate the potential further. Therefore, we consider an AM resupply lead time of about one day $\left(\mu_{A}=25\right)$ and a MTBF of 5 months $\left(\lambda_{A}=0.2\right)$. The CM part follows the characteristics of Item $3\left(\lambda_{C}=0.01, \mu_{C}=0.15, c_{C}=10\right)$. The other parameter values remain unchanged. Even under these conditions, dual sourcing leads to cost savings of about $17 \%$ on average. We conclude that the application of AM at remote locations deserves more attention in the literature.

The cost savings with dual sourcing become larger when increasing the holding costs fraction $(\kappa)$, decreasing the maintenance costs $(m)$, and decreasing the installed base size $(k)$, cf. column 'Cost savings compared to Best' in Table 2. If we study the impact of changing these values on the cost composition in Fig. 6, the underlying reasons become clear. In case of parameter $m$, the holding and the backorder costs decrease as a percentage of the total costs while the maintenance costs increase significantly. Hence, the cost saving potential of dual sourcing reduces. We find a similar result for the installed base size $(k)$, except that also the purchasing costs 
Fig. 6 Percentage point change of the cost factors if parameter value is increased from its minimum value to its maximum value
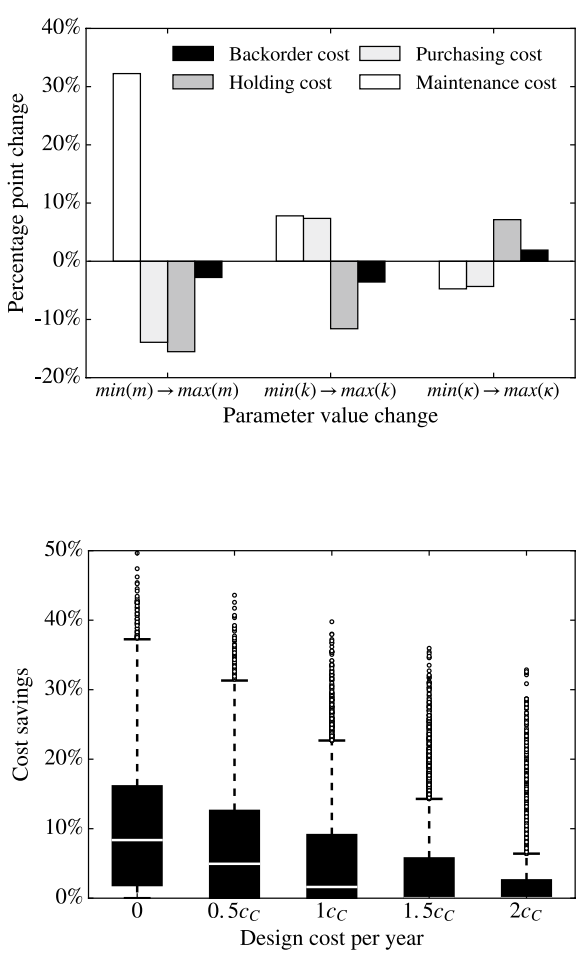

Fig. 7 Effect of design costs on the cost savings with dual sourcing compared to single sourcing with CM

increase as a percentage of the total costs. Note that the installed base size primarily influences the demand rate and thus explains the increase in the purchasing costs. In contrast, an increase in the holding costs fraction $(\kappa)$ increases the holding costs and the backorder costs while the maintenance and purchasing costs decrease.

As discussed in Sect. 3.7, designing one instead of two part versions typically causes additional design costs. In Fig. 7, we show the effect of additional design costs on the average cost savings with dual sourcing compared to single sourcing with $\mathrm{CM}$. Here, the assessed values for the additional design costs are in the same order of magnitude as we find in the case study presented in Sect. 5. As expected, the value of a dual sourcing approach decreases with increasing design costs. Nevertheless, even in case of high design costs we still find various instances where dual sourcing offers cost savings of more than $20 \%$ compared to single sourcing with CM in Fig. 7. Furthermore, technological advancements of AM are likely to decrease design cost in the future.

\subsection{Summary of key findings}

The numerical experiments lead to the following conclusions:

1. With dual sourcing, it is possible to exploit most benefits of a typically short AM resupply lead time while common drawbacks of high AM piece price and/or low AM part reliability have a significantly lower impact than with AM single sourc- 
ing. For example, even if the AM piece price $\left(c_{A}\right)$ or the AM failure rate $\left(\lambda_{A}\right)$ is up to three times higher than those of the CM part, we still find instances with cost savings of more than $30 \%$ compared to the best single sourcing approach.

2. Printing spare parts on demand do not appear to be suitable for downtime critical spare parts. Instead, stock remains necessary to reduce the risk of expensive downtime. As a result, dual sourcing largely outperforms single sourcing with AM. On average, we find cost savings of more than $48 \%$ even if the AM resupply lead time is more than twenty times shorter than the CM resupply lead time.

3. In line with the dual sourcing literature, AM typically functions as an emergency source. In most cases, more than $80 \%$ of the base stock has to be depleted before we order the first time from the AM source. In case both item versions are in stock, the CM item is usually used first. This result is a consequence of the typically low CM failure rate.

4. If single sourcing with $\mathrm{CM}$ leads to high holding and backorder costs relative to the other cost factors, dual sourcing is most promising. This type of conditions is typically obtained with a low CM resupply rate $\left(\mu_{C}\right)$, a high holding costs fraction $(\kappa)$, a high backorder costs $(b)$, low maintenance costs, and/or a low demand rate.

\section{Case study}

To gain further insights into the practical implications of combining AM and CM sourcing modes, we conduct a case study at a service provider in the aerospace industry. More explicitly, we consider a hinge bracket that is used for connecting the rudder and the aircraft. According to the Federal Aviation Administration (FAA), the hinge is categorized as a Class 2 product. That is, a failure may jeopardize the safety of an aircraft and thus is considered critical. To avoid devastating consequences, aircraft manufacturers typically work with redundancies. Nevertheless, a failure of a hinge has to be corrected upon discovery. Hence, from an operational perspective, redundancies only exist to prevent failure risks during a flight. But during maintenance, there are no redundancies. The most common failure modes are fractures of the hinge that are caused by fatigue, tensile stress, or corrosion.

Figure 8 illustrates the $\mathrm{CM}$ and AM design of the hinge. The service provider estimated the total design costs for the AM hinge at around 15,000 euro. While the $\mathrm{CM}$ hinge is an aluminum machined part, the AM hinge is built from titanium powder (Ti-6AL-4V) with Selective Laser Melting (SLM). Next to accounting for different material properties, the AM design is topologically optimized to reduce the weight of the hinge. Overall, despite titanium being heavier than aluminum, the topology optimization leads to a weight reduction of about $25 \%$, which leads to fuel savings of approximately $o_{A}=15$ euro per AM part per year.

According to further investigations reported by the service provider, cost reductions resulting from fuel savings though were not sufficient to justify a transition to AM. Today's unstandardized AM certification process in the aerospace industry for structural parts would require an investment of about 250,000 euro which renders any application of AM economically infeasible. However, the service provider is confident that fast regulatory process development may dismantle the certification 
costs barrier soon. For instance, the FAA just distributed a draft version of its Additive Manufacturing Strategic Roadmap to establish a standardized certification process (3ders.org 2017).

Another concern constitutes the high AM piece price. The service provider argues that the AM piece price has to decrease significantly before the production with AM methods becomes economically feasible. We provide the input parameters for the hinge case in Table 3. If not mentioned otherwise below, the parameter values were obtained from company records.

For the design costs, we assume that the costs are evenly spread over the remaining life cycle of 15 years and thus encounter depreciation costs $d=1000$ euro per year. Compared to the numerical experiments, we take operational cost savings into account by following the explanation in Sect. 3.7.

The failure rate $\lambda_{A}$ is based on the following insights: mechanical tests revealed that the AM hinge exhibits superior static strength compared to the CM hinge. Also, given that the AM hinge is produced with titanium powder rather than with aluminum, failures caused by corrosion can be ruled out. On the other hand, the surface roughness and porosity expected from the SLM production process may concentrate more tensions in the hinge. Hence, it is likely that the AM hinge fails more often under cyclic load caused by fatigue. Combining these different observations, AM experts estimate $\lambda_{A}=0.75 \lambda_{C}$. Note that from a regulatory perspective even inferior reliability would be acceptable as long as it is possible to prove that the integrity of the system is not compromised and that airworthiness is not affected. For the hinge bracket, these criteria are met given the use of redundancy, regular inspections, and the favorable failure behavior.

The backorder costs $b$ follow from the criticality of the hinge. According to company representatives, it is likely that a stock-out leads to additional downtime of the aircraft. Hence, depending on the aircraft type, backorder costs $b$ vary between 15,000 and 50,000 euro per day. For our analysis, we use $b=32,500$ as a base case but note that our results are not very sensitive to changes of $b$ in that range: the longrun average service cost per year differs by less than $1 \%$ if we compare $b=15,000$ and $b=50,000$.

Currently, the case company serves an installed base of $k=382$ hinges, each with a mean time between failure of 27.78 years. To obtain computationally tractable

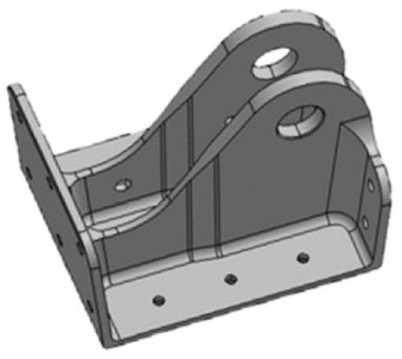

(a)

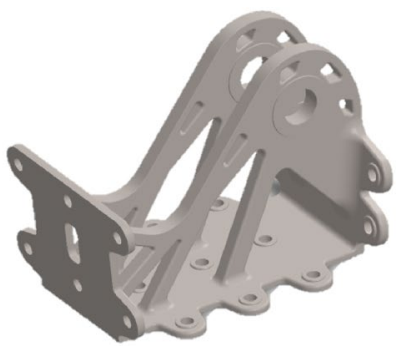

(b)

Fig. $8 \mathrm{CM}$ (a) and AM hinge (b) design 
data, we need to aggregate demand streams. To that end, we consider a smaller $k^{\prime}$ instead and multiply the failure rates $\lambda_{A}$ and $\lambda_{C}$ with the fraction $382 / k^{\prime}$ in order to keep the total demand rate unchanged. Given that, as soon as $k^{\prime}$ is sufficiently large, the demand variability becomes nearly independent of the installed base size, this transformation leads to proportionally the same results. After the results are computed, we simply multiply the operational cost savings with $382 / k^{\prime}$ to obtain the savings for the case $k=382$. We illustrate this effect by plotting the long-run average net cost (cost minus savings) per year with dual sourcing for different values of $k^{\prime}$ in Fig. 9. In the remainder, we use $k^{\prime}=50$ for our analysis.

Figure 10 shows the total cost and savings for the three sourcing options. Note that here we assumed that the AM source is always prepared for the dual sourcing mode. Hence, as is the case for the hinge bracket, dual sourcing may lead to higher cost than single sourcing with $\mathrm{CM}$. In particular, we observe that dual sourcing leads to total costs of about 13,838 euro. Even if we were considering that with dual sourcing we can secure cost savings of about 4581 euro from weight reductions, using CM only remains the better choice. An explanation for this results offer the high purchasing costs. As we found in the numerical experiments (cf. Sect. 4), dual sourcing is most valuable if the holding or the backorder costs dominate. For the hinge bracket, this is clearly not the case since we only find 70 euro (AM only), 55 euro (Dual), and 120 euro (CM only) for the backorder costs, and 752 euro (AM only), 507 euro (Dual), and 534 euro (CM only) for the holding costs.

To that end, it appears likely that the value of AM for the hinge bracket case (if any) would originate from operational rather than service cost savings. Yet, it remains an open research question if and how a dual sourcing approach is useful for such scenarios. The results clearly indicate a trade-off between operational cost savings and service cost. That is, in order to secure higher operational cost savings, we typically would install more AM parts than is optimal to minimize service cost.

Finally, and this represents a key learning for us from this case study, we emphasize that today's attention for AM technologies is mainly motivated by the prospect of design improvements/operational cost savings. While this certainly has its value, it

Table 3 Model input data hinge case

\begin{tabular}{lll}
\hline Parameter & Value & Unit \\
\hline$c_{A}$ & 1197 & Euro/unit \\
$c_{C}$ & 480 & Euro/unit \\
$\mu_{A}$ & 12 & Supplies/year \\
$\mu_{C}$ & 4 & Supplies/year \\
$\lambda_{A}$ & 0.027 & Failures/year \\
$\lambda_{C}$ & 0.036 & Failures/year \\
$k$ & 382 & Installed parts \\
$b$ & 32,500 & Euro/day \\
$m$ & 100 & Euro/failure \\
$d$ & 1000 & Euro/year \\
$o_{A}$ & 15 & Euro/AM part/year \\
$\kappa$ & 0.2 & - \\
\hline
\end{tabular}


Fig. 9 Effect of consolidating demand streams where $\lambda_{A}=382 / k \lambda_{A}$ and $\lambda_{C}=382 / k \lambda_{C}$

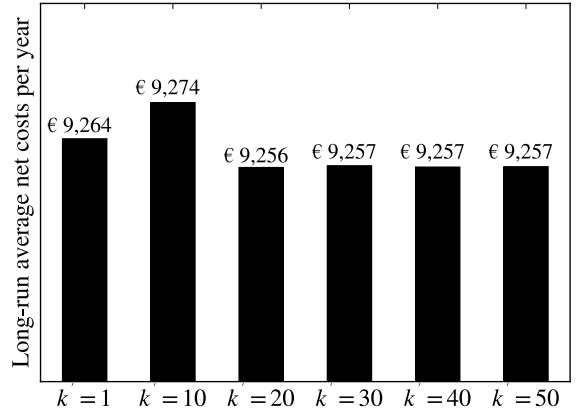

Fig. 10 Total cost and savings for the three sourcing options excluding certification costs

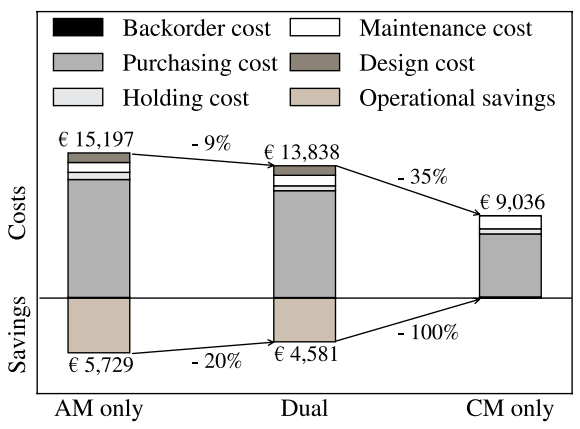

dilutes the contribution of AM technologies to service cost savings. In our opinion, there are two reasons for this: first, design improvements often scale with quantity. For example, if topology optimization leads to weight reductions of a few grams only, the effort is typically justified by a large installed base size. Accordingly, AM demonstrators frequently exhibit 'high' demand rates (say, >10/year) rather than high holding and backorder costs. So, as also exemplified by the hinge bracket, service cost savings become less interesting. Second, the prospect of design improvements and technological complexity of AM methods typically put engineers in the lead to identify parts worthy of production with AM methods. Unfortunately, as a result, logistic opportunities may be underrated/overlooked. Instead, logisticians should become more involved in this task. To that end, we believe that it is important to carry out more case-based research in the low-volume, downtime critical spare parts business to exemplify the merit of AM methods in this field to decision makers.

\section{Conclusion}

Demand fulfillment with a mix of $\mathrm{AM}$ and $\mathrm{CM}$ production methods has not been sufficiently discussed in the literature yet. In this paper, we have addressed this gap by studying a dual sourcing concept where AM and CM methods are used in parallel to fulfill spare parts demand. A novel aspect of our model is that we account for the different failure behavior of parts obtained from AM and CM supply sources which influences future demand. 
Overall, dual sourcing turns out to reinforce the value of AM methods in the spare parts business. In particular, this holds true if backorder costs and holding costs are high relative to purchasing and maintenance costs. Moreover, our results stipulate that the concept of printing spare parts on demand usually is not suitable for downtime critical parts. Instead, stock remains necessary even if the AM resupply lead time is very short. In our opinion, the most remarkable finding, however, is that dual sourcing offers an approach to profit from the fast AM resupply lead time even if the AM piece price is high and/or AM part reliability is low compared to the CM part. Consequently, dual sourcing may extend the operating range of AM methods in the spare parts business significantly.

In the light of this finding, new sourcing concepts are likely. For instance, our results indicate that supplementing CM supply with less reliable, but locally producible AM parts may reduce operating costs of capital goods at remote locations considerably. It appears valuable to investigate the effect of this approach more in detail. Furthermore, one may consider scenarios where the availability of the CM source is restricted. For example, it seems realistic to assume that supply risks are higher for the CM source due to the digital nature of AM production methods. Another model extension may consider a typically higher uncertainty about the AM failure behavior. While for the CM source, we typically (should) know historical failure records, the failure behavior of AM items is still rather unclear. Finally, in this paper, we assumed a base stock policy. However, state-dependent demand rates may deem a dynamic inventory policy optimal. The model extension proposed in Sect. 3.6 may serve future research as a starting point for further investigations on this matter.

Acknowledgements This research is part of the project 'Sustainability Impact of New Technology on After sales Service supply chains (SINTAS)' and has been sponsored by the Netherlands Organization for Scientific Research under Project Number 438-13-207.

Open Access This article is licensed under a Creative Commons Attribution 4.0 International License, which permits use, sharing, adaptation, distribution and reproduction in any medium or format, as long as you give appropriate credit to the original author(s) and the source, provide a link to the Creative Commons licence, and indicate if changes were made. The images or other third party material in this article are included in the article's Creative Commons licence, unless indicated otherwise in a credit line to the material. If material is not included in the article's Creative Commons licence and your intended use is not permitted by statutory regulation or exceeds the permitted use, you will need to obtain permission directly from the copyright holder. To view a copy of this licence, visit http://creativecommons.org/licen ses/by/4.0/.

\section{Appendix 1: Exponentially distributed resupply rate}

In order to gain insights on how the replenishment variability influences the results, we conducted a simulation study. Figures 11 and 12 show the effect of an increasing replenishment variability on the cost for two different instances. As we observe, the effect is limited and thus is in line with the findings in the literature, cf. Sect. 3.1.

The parameter settings for both cases are in the same range as for the numerical experiments discussed in Sect. 4. In particular, for the first instance (Fig. 11) we use following parameter settings: $k=20$ installed parts, $b=2000$ euro/month, 
Fig. 11 Simulation results for different variabilities of the replenishment lead time

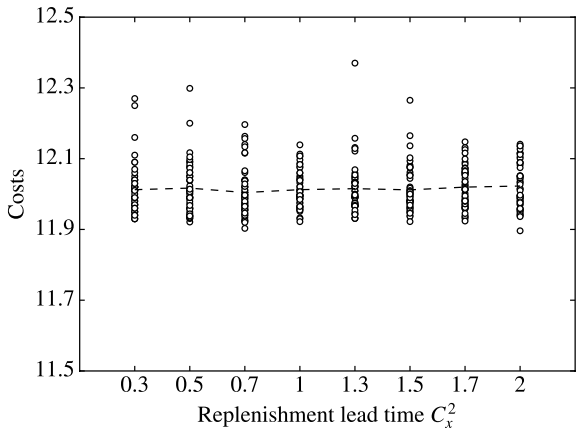

Fig. 12 Simulation results for different variabilities of the replenishment lead time

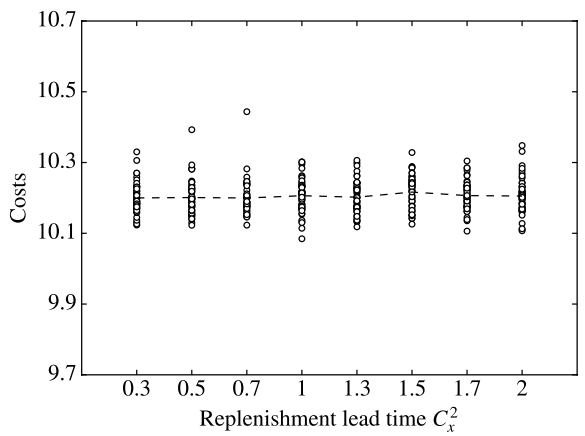

$\lambda_{A}=0.035$ failures/month, $\mu_{C}=0.15$ supplies/month, $c_{C}=10$ euro/part, $m=10$ euro/failure, $\kappa=0.25, c_{A}=20$ euro/part, $\lambda_{C}=0.01$ failures/month, and $\mu_{A}=4$ supplies/month. For the second instance (Fig. 12), we use: $k=30$ installed parts, $b=200$ euro/month, $\lambda_{A}=0.035$ failures $/$ month, $\mu_{C}=0.15$ supplies $/$ month, $c_{C}=10$ euro/part, $m=2$ euro/failure, $\kappa=0.25, c_{A}=20$ euro/part, $\lambda_{C}=0.01$ failures/month, and $\mu_{A}=4$ supplies/month.

\section{Appendix 2: Balance equations basic model}

Subsequently, we present the balance equations in which $p_{0}$ refers to the state probability under consideration. For example, in case $x_{2}\left(n_{C, \mathbf{i}}, n_{A, \mathbf{i}}+1, r_{C, \mathbf{i}}, r_{A, \mathbf{i}}-1, s_{C, \mathbf{i}}, s_{A, \mathbf{i}}\right)$, then $p_{\bullet}=p_{\left(n_{C, \mathrm{i}}, n_{A, \mathrm{i}}+1, r_{C, \mathrm{i}}, r_{A, \mathrm{i}}-1, s_{C, \mathrm{i}}, s_{A, \mathrm{i}}\right)}$. Furthermore, if the maintenance decision is indifferent (i.e., no stock available), we use $x_{2}(\mathbf{i})$ and $x_{4}(\mathbf{i})$ as default. 


$$
\begin{aligned}
\left(\lambda_{C} n_{C, \mathbf{i}}\right. & \left.+\lambda_{A} n_{A, \mathbf{i}}+\mu_{C} r_{C, \mathbf{i}}+\mu_{A} r_{A, \mathbf{i}}\right) p_{\mathbf{i}} \\
= & \lambda_{A}\left(n_{A, \mathbf{i}}+1\right) x_{2}\left(n_{C, \mathbf{i}}, n_{A, \mathbf{i}}+1, r_{C, \mathbf{i}}, r_{A, \mathbf{i}}-1, s_{C, \mathbf{i}}, s_{A, \mathbf{i}}\right) p . \\
& +\lambda_{A} n_{A, \mathbf{i}} x_{1}\left(n_{C, \mathbf{i}}, n_{A, \mathbf{i}}, r_{C, \mathbf{i}}, r_{A, \mathbf{i}}-1, s_{C, \mathbf{i}}, s_{A, \mathbf{i}}+1\right) p . \\
& +\lambda_{A}\left(n_{A, \mathbf{i}}+1\right) x_{2}\left(n_{C, \mathbf{i}}-1, n_{A, \mathbf{i}}+1, r_{C, \mathbf{i}}, r_{A, \mathbf{i}}-1, s_{C, \mathbf{i}}+1, s_{A, \mathbf{i}}\right) p . \\
& +\lambda_{A}\left(n_{A, \mathbf{i}}+1\right) x_{4}\left(n_{C, \mathbf{i}}, n_{A, \mathbf{i}}+1, r_{C, \mathbf{i}}-1, r_{A, \mathbf{i}}, s_{C, \mathbf{i}}, s_{A, \mathbf{i}}\right) p . \\
& +\lambda_{A} n_{A, \mathbf{i}} x_{3}\left(n_{C, \mathbf{i}}, n_{A, \mathbf{i}}, r_{C, \mathbf{i}}-1, r_{A, \mathbf{i}}, s_{C, \mathbf{i}}, s_{A, \mathbf{i}}+1\right) p . \\
& +\lambda_{A}\left(n_{A, \mathbf{i}}+1\right) x_{4}\left(n_{C, \mathbf{i}}-1, n_{A, \mathbf{i}}+1, r_{C, \mathbf{i}}-1, r_{A, \mathbf{i}}, s_{C, \mathbf{i}}+1, s_{A, \mathbf{i}}\right) p . \\
& +\lambda_{C}\left(n_{C, \mathbf{i}}+1\right) x_{2}\left(n_{C, \mathbf{i}}+1, n_{A, \mathbf{i}}, r_{C, \mathbf{i}}, r_{A, \mathbf{i}}-1, s_{C, \mathbf{i}}, s_{A, \mathbf{i}}\right) p . \\
& +\lambda_{C}\left(n_{C, \mathbf{i}}+1\right) x_{1}\left(n_{C, \mathbf{i}}+1, n_{A, \mathbf{i}}-1, r_{C, \mathbf{i}}, r_{A, \mathbf{i}}-1, s_{C, \mathbf{i}}, s_{A, \mathbf{i}}+1\right) p . \\
& +\lambda_{C} n_{C, \mathbf{i}} x_{2}\left(n_{C, \mathbf{i}}, n_{A, \mathbf{i}}, r_{C, \mathbf{i}}, r_{A, \mathbf{i}}-1, s_{C, \mathbf{i}}+1, s_{A, \mathbf{i}}\right) p . \\
& +\lambda_{C}\left(n_{C, \mathbf{i}}+1\right) x_{4}\left(n_{C, \mathbf{i}}+1, n_{A, \mathbf{i}}, r_{C, \mathbf{i}}-1, r_{A, \mathbf{i}}, s_{C, \mathbf{i}}, s_{A, \mathbf{i}}\right) p . \\
& +\lambda_{C}\left(n_{C, \mathbf{i}}+1\right) x_{3}\left(n_{C, \mathbf{i}}+1, n_{A, \mathbf{i}}-1, r_{C, \mathbf{i}}-1, r_{A, \mathbf{i}}, s_{C, \mathbf{i}}, s_{A, \mathbf{i}}+1\right) p . \\
& +\lambda_{C} n_{C, \mathbf{i}} x_{4}\left(n_{C, \mathbf{i}}, n_{A, \mathbf{i}}, r_{C, \mathbf{i}}-1, r_{A, \mathbf{i}}, s_{C, \mathbf{i}}+1, s_{A, \mathbf{i}}\right) p . \\
& +\mu_{A}\left(r_{A, \mathbf{i}}+1\right) p_{\left(n_{C, \mathbf{i}}, n_{A, \mathbf{i}}, r_{C, \mathbf{i}}, r_{A, \mathbf{i}}+1, s_{C, \mathbf{i}}, s_{A, \mathbf{i}}-1\right)} \\
& +\mu_{A}\left(r_{A, \mathbf{i}}+1\right) p_{\left(n_{C, \mathbf{i}}-1, n_{A, \mathbf{i}}, r_{C, \mathbf{i}}, r_{A, \mathbf{i}}+1, s_{C, \mathbf{i}}, s_{A, \mathbf{i}}\right)} \\
& +\mu_{C}\left(r_{C, \mathbf{i}}+1\right) p_{\left(n_{C, \mathbf{i}}, n_{A, \mathbf{i}}, r_{C, \mathbf{i}}+1, r_{A, \mathbf{i}}, s_{C, \mathbf{i}}-1, s_{A, \mathbf{i}}\right)} \\
& +\mu_{C}\left(r_{C, \mathbf{i}}+1\right) p_{\left(n_{C, \mathbf{i}}-1, n_{A, \mathbf{i}}, r_{C, \mathbf{i}}+1, r_{A, \mathbf{i}}, s_{C, \mathbf{i}}, s_{A, \mathbf{i}}\right)}
\end{aligned}
$$

\section{Appendix 3: Joint optimization}

To jointly optimize the base stock level, maintenance policy, and sourcing policy, we increase the decision space of the sourcing decision and add the possibility to order nothing upon failure of an item. This extension enables the transition to states where $N$ decreases. Accordingly, we obtain the following updated decision space:

$$
\begin{aligned}
\Omega=\{ & \left(n_{C, \mathbf{i}}, n_{A, \mathbf{i}}, r_{C, \mathbf{i}}, r_{A, \mathbf{i}}, s_{C, \mathbf{i}}, s_{A, \mathbf{i}}\right): \\
& L B \geq N \leq U B \\
& n_{C, \mathbf{i}}+n_{A, \mathbf{i}}+r_{C, \mathbf{i}}+r_{A, \mathbf{i}}+s_{C, \mathbf{i}}+s_{A, \mathbf{i}}=N \\
& s_{C, \mathbf{i}}+s_{A, \mathbf{i}}=\max \left\{N-k-r_{C, \mathbf{i}}-r_{A, \mathbf{i}} ; 0\right\} \\
& n_{C, \mathbf{i}}+n_{A, \mathbf{i}} \leq k \\
& \left.n_{C, \mathbf{i}}, n_{A, \mathbf{i}}, r_{C, \mathbf{i}}, r_{A, \mathbf{i}}, s_{C, \mathbf{i}}, s_{A, \mathbf{i}} \geq 0\right\}
\end{aligned}
$$

where $L B$ and $U B$ describe the lower and upper bound on $N$, respectively. We set $L B=k$ which permits the extreme case to manufacture parts on demand only. In case of the $U B$, we compute the optimal base stock level $\hat{S}$ of a single sourcing 


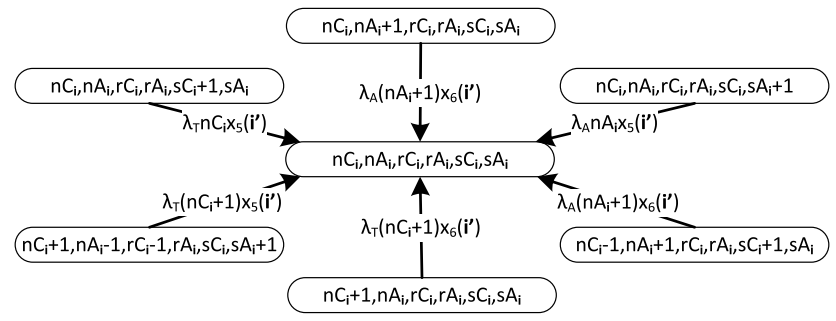

Fig. 13 Additional transitions for state $\left(n_{C, \mathbf{i}}, n_{A, \mathbf{i}}, r_{C, \mathbf{i}}, r_{A, \mathbf{i}}, s_{C, \mathbf{i}}, s_{A, \mathbf{i}}\right)$ for joint optimization

model with $\lambda=\max \left\{\lambda_{C} ; \lambda_{A}\right\}, \mu=\min \left\{\mu_{C} ; \mu_{A}\right\}$ and $c=\min \left\{c_{C} ; c_{A}\right\}$. Next, we set $U B=k+\hat{S}$. As this is a worst case scenario, we always find an upper bound, even though it may not be very tight. An alternative is to use as a heuristic upper bound which is equal to the base stock level of the better performing single sourcing option. Unfortunately, our numerical experiments reveal that this bound is not always sufficient. In this case, we set $S=U B-k$ and follow the greedy heuristic as explained in Sect. 3.5.

To include the decision option to order nothing, we increase the action space of the sourcing decision. Accordingly, we add decision options:

- $c=5$ : take AM version from stock (if possible), and order nothing.

- $c=6$ : take CM version from stock (if possible), and order nothing.

The increase in the action space leads to six additional transitions. These are illustrated for state $\left(n_{C, \mathbf{i}}, n_{A, \mathbf{i}}, r_{C, \mathbf{i}}, r_{A, \mathbf{i}}, s_{C, \mathbf{i}}, s_{A, \mathbf{i}}\right)$ in Fig. 13.

\section{Appendix 4: Dynamic inventory policy}

Given that the part is available with two versions of different reliabilities, the expected number of failures is state dependent. Same holds for the expected number of arrivals given the difference in resupply rate. As a consequence, the stocking policy might depend on the state. For example, consider the situation where the installed base is mainly equipped with AM items. In this case, a higher failure frequency is likely, and thus, we may want to increase the base stock level to avoid backorder costs. Then, if the number of $\mathrm{CM}$ components increases, we may find that it is cost efficient to reduce the base stock level to decrease holding costs.

Given that we already added the possibility to reduce $N$ (cf. Appendix 2), we only need to include transitions that allow increasing $N$ to facilitate a dynamic base stock level. 
Again, we realize this extension by increasing the action space to allow ordering more than one part. Given that typically $\lambda_{C}<\lambda_{A}$, the possibility to order more than one part is most valuable if a CM part fails. Also, it is not reasonable to order more than 2 parts because this would imply that it would have been useful to already order 2 parts at a previous failure of a CM part. Accordingly, we add six decision options:

- $c=7$ : take CM part from stock (if possible), and order AM and CM part.

- $c=8$ : take CM part from stock (if possible), and order 2 AM parts.

- $c=$ 9: take AM part from stock (if possible), and order AM and CM part.

- $c=10$ : take AM part from stock (if possible), and order 2 AM parts.

- $c=11$ : take CM part from stock (if possible), and order 2 CM parts.

- $c=12$ : take AM part from stock (if possible), and order $2 \mathrm{CM}$ parts.

We can incorporate these actions by regarding nine additional transitions. These are illustrated for state $\left(n_{C, \mathbf{i}}, n_{A, \mathbf{i}}, r_{C, \mathbf{i}}, r_{A, \mathbf{i}}, s_{C, \mathbf{i}}, s_{A, \mathbf{i}}\right)$ in Fig. 14.

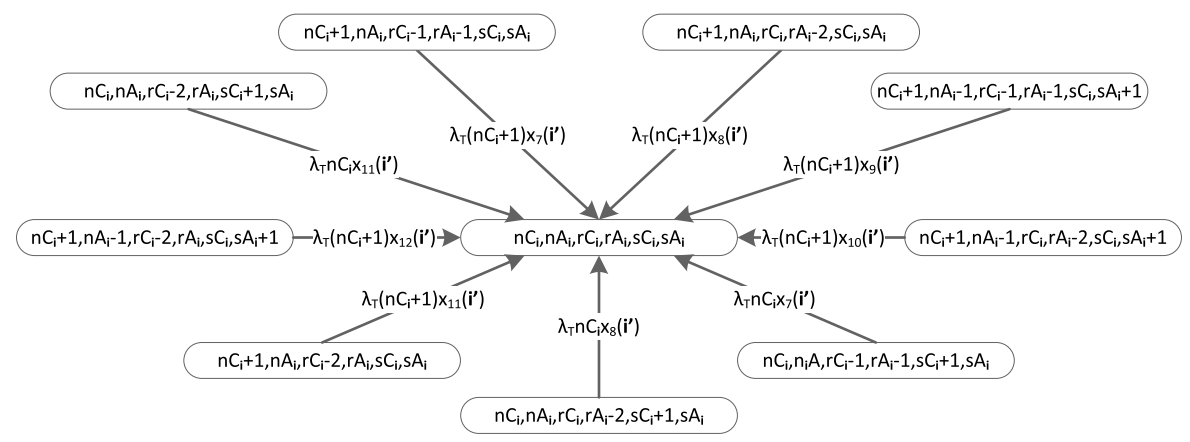

Fig. 14 Additional transitions for state $\left(n_{C, \mathbf{i}}, n_{A, \mathbf{i}}, r_{C, \mathbf{i}}, r_{A, \mathbf{i}}, s_{C, \mathbf{i}}, s_{A, \mathbf{i}}\right)$ for dynamic inventory policy 


\section{Appendix 5: Supplementary results numerical experiments}

\section{(See Table 4)}

Table 4 Minimum and maximum values over corresponding instances, where single sourcing with only $\mathrm{CM}$ is denoted by $C M$, single sourcing with only AM by $A M$, single sourcing with the best instance specific approach by Best, and dual sourcing by Dual

\begin{tabular}{|c|c|c|c|c|c|c|c|c|c|c|c|}
\hline \multirow{3}{*}{$\begin{array}{l}\text { Param- } \\
\text { eter }\end{array}$} & \multirow[t]{3}{*}{ Value } & \multirow[t]{3}{*}{ Unit } & \multirow{2}{*}{\multicolumn{3}{|c|}{$\begin{array}{l}\text { Maximum savings } \\
\text { compared to }\end{array}$}} & \multirow{2}{*}{\multicolumn{3}{|c|}{$\frac{\text { Minimum base }}{\text { stock level }}$}} & \multirow{2}{*}{\multicolumn{3}{|c|}{$\begin{array}{l}\text { Maximum base } \\
\text { stock level }\end{array}$}} \\
\hline & & & & & & & & & & & \\
\hline & & & $\mathrm{CM}$ & $\mathrm{AM}$ & Best & $\mathrm{CM}$ & AM & Dual & $\mathrm{CM}$ & AM & Dual \\
\hline & 1 & - & $45 \%$ & $82 \%$ & $21 \%$ & 1 & 0 & 0 & 6 & 7 & 6 \\
\hline \multirow[t]{3}{*}{ Item } & 2 & - & $50 \%$ & $84 \%$ & $29 \%$ & 2 & 0 & 0 & 7 & 7 & 7 \\
\hline & 3 & - & $47 \%$ & $86 \%$ & $35 \%$ & 2 & 0 & 0 & 8 & 7 & 7 \\
\hline & 10 & Installed parts & $50 \%$ & $82 \%$ & $35 \%$ & 1 & 0 & 0 & 4 & 4 & 4 \\
\hline \multirow[t]{3}{*}{$k$} & 20 & Installed parts & $43 \%$ & $85 \%$ & $35 \%$ & 2 & 0 & 0 & 6 & 5 & 5 \\
\hline & 30 & Installed parts & $41 \%$ & $86 \%$ & $35 \%$ & 3 & 0 & 1 & 8 & 7 & 7 \\
\hline & 0.0175 & Failures/month & $50 \%$ & $65 \%$ & $34 \%$ & 1 & 0 & 0 & 8 & 4 & 6 \\
\hline \multirow[t]{3}{*}{$\lambda_{A}$} & 0.035 & Failures/month & $41 \%$ & $80 \%$ & $35 \%$ & 1 & 0 & 1 & 8 & 6 & 7 \\
\hline & 0.0525 & Failures/month & $35 \%$ & $86 \%$ & $35 \%$ & 1 & 0 & 1 & 8 & 7 & 7 \\
\hline & 1 & Supplies/month & $29 \%$ & $86 \%$ & $23 \%$ & 1 & 0 & 1 & 8 & 7 & 7 \\
\hline \multirow[t]{3}{*}{$\mu_{A}$} & 2 & Supplies/month & $40 \%$ & $86 \%$ & $31 \%$ & 1 & 0 & 0 & 8 & 5 & 6 \\
\hline & 4 & Supplies/month & $50 \%$ & $85 \%$ & $35 \%$ & 1 & 0 & 0 & 8 & 3 & 6 \\
\hline & 10 & Euro/part & $50 \%$ & $73 \%$ & $35 \%$ & 1 & 0 & 0 & 8 & 7 & 7 \\
\hline \multirow[t]{3}{*}{$c_{A}$} & 20 & Euro/part & $34 \%$ & $81 \%$ & $34 \%$ & 1 & 0 & 0 & 8 & 6 & 7 \\
\hline & 30 & Euro/part & $30 \%$ & $86 \%$ & $30 \%$ & 1 & 0 & 1 & 8 & 6 & 7 \\
\hline & 20 & Euro/month & $50 \%$ & $86 \%$ & $33 \%$ & 1 & 0 & 0 & 4 & 4 & 4 \\
\hline \multirow[t]{3}{*}{$b$} & 200 & Euro/month & $45 \%$ & $85 \%$ & $33 \%$ & 2 & 1 & 1 & 6 & 5 & 6 \\
\hline & 2000 & Euro/month & $47 \%$ & $84 \%$ & $35 \%$ & 3 & 1 & 1 & 8 & 7 & 7 \\
\hline & 2 & Euro/failure & $50 \%$ & $86 \%$ & $35 \%$ & 1 & 0 & 0 & 8 & 7 & 7 \\
\hline \multirow[t]{3}{*}{$m$} & 10 & Euro/failure & $43 \%$ & $85 \%$ & $34 \%$ & 1 & 0 & 0 & 8 & 7 & 7 \\
\hline & 18 & Euro/failure & $38 \%$ & $85 \%$ & $28 \%$ & 1 & 0 & 0 & 8 & 7 & 7 \\
\hline & 0.15 & - & $45 \%$ & $86 \%$ & $34 \%$ & 1 & 0 & 0 & 8 & 7 & 7 \\
\hline \multirow[t]{2}{*}{$\kappa$} & 0.2 & - & $46 \%$ & $85 \%$ & $34 \%$ & 1 & 0 & 0 & 8 & 7 & 7 \\
\hline & 0.25 & - & $50 \%$ & $83 \%$ & $35 \%$ & 1 & 0 & 0 & 7 & 6 & 6 \\
\hline
\end{tabular}

\section{References}

3dersorg (2017) FAA's 8-year 'Additive Manufacturing strategic roadmap' lays groundwork for 3D printed aircraft development. https://www.3ders.org/articles/20171023-faas-8-year-additive-manuf acturing-strategic-roadmap-lays-groundwork-for-3d-printed-aircraft-development.html

Airbus (2016) Innovative 3D printing solutions are "taking shape" within Airbus. http://www.airbus.com/ newsevents/news-events-single/detail/innovative-3d-printing-solutions-are-taking-shape-withi n-airbus/ 
Alfredsson P, Verrijdt J (1999) Modeling emergency supply flexibility in a Two-Echelon inventory system. Manag Sci 45(10):1416-1431. https://doi.org/10.1287/mnsc.45.10.1416

Allon G, Van Mieghem JA (2010) Global Dual Sourcing: Tailored Base-Surge Allocation to Near- and Offshore production. Manag Sci 56(1):110-124. https://doi.org/10.1287/mnsc.1090.1099

Alvarez E, van der Heijden M, Zijm W (2013) The selective use of emergency shipments for servicecontract differentiation. Int J Prod Econ 143(2):518-526. https://doi.org/10.1016/j.ijpe.2012.02.019

Arts J, van Vuuren M, Kiesmüller G (2011) Efficient optimization of the dual-index policy using Markov chains. IIE Trans 43(8):604-620. https://doi.org/10.1080/0740817X.2010.550908

Axsäter S (2007) A heuristic for triggering emergency orders in an inventory system. Eur J Oper Res 176(2):880-891. https://doi.org/10.1016/j.ejor.2005.09.002

Axsäter S (2014) An improved decision rule for emergency replenishments. Int J Prod Econ 157(1):313317. https://doi.org/10.1016/j.ijpe.2014.01.021

Barankin EW (1961) A delivery-lag inventory model with an emergency provision (the single-period case). Naval Res Logist Q 8(3):285-311. https://doi.org/10.1002/nav.3800080310

Basten RJ (2010) Designing logistics support systems: level of repair analysis and spare parts inventories. Ph.D. thesis, University of Twente, Enschede, The Netherlands, https://doi.org/10.3990/1.97890 36529679

Book TA, Sangid MD (2016) Evaluation of select surface processing techniques for in situ application during the additive manufacturing build process. JOM 68(7):1780-1792. https://doi.org/10.1007/ s11837-016-1897-y

Campbell T, Williams C, Ivanova O, Garrett B (2012) Could 3D Printing Change the World?. Tech. rep, Atlantic Council

de Jonge B, Scarf PA (2019) A review on maintenance optimization. Eur J Oper Res. https://doi. org/10.1016/j.ejor.2019.09.047

Eyers DR, Potter AT (2017) Industrial additive manufacturing: a manufacturing systems perspective. Comput Ind 92-93:208-218. https://doi.org/10.1016/j.compind.2017.08.002

Frazier WE (2014) Metal additive manufacturing: a review. J Mater Eng Perform 23(6):1917 (1928), https://doi.org/10.1007/s11665-014-0958-Z

Godoy DR, Pascual R, Knights P (2014) A decision-making framework to integrate maintenance contract conditions with critical spares management. Reliab Eng Syst Saf 131:102-108. https://doi. org/10.1016/j.ress.2014.06.022

Holmström J, Partanen J, Tuomi J, Walter M (2010) Rapid manufacturing in the spare parts supply chain. J Manuf Technol Manag 21(6):687-697. https://doi.org/10.1108/17410381011063996

Holweg M (2015) The limits of 3-D printing. Harvard Business Review June:2-4, https://hbr. org/2015/06/the-limits-of-3d-printing

Hu Q, Boylan JE, Chen H, Labib A (2018) OR in spare parts management: a review. Eur J Oper Res 266(2):395-414. https://doi.org/10.1016/j.ejor.2017.07.058

Huang S, Axsäter S, Dou Y, Chen J (2011) A real-time decision rule for an inventory system with committed service time and emergency orders. Eur J Oper Res 215(1):70-79. https://doi.org/10.1016/j. ejor.2011.05.029

Khajavi SH, Partanen J, Holmström J (2014) Additive manufacturing in the spare parts supply chain. Computers in Industry 65(1)

Kiesmüller G (2003) A new approach for controlling a hybrid stochastic manufacturing/remanufacturing system with inventories and different leadtimes. Eur J Oper Res 147(1):62-71. https://doi. org/10.1016/S0377-2217(02)00351-X

Knofius N, van der Heijden M, Zijm W (2018) Consolidating spare parts for asset maintenance with additive manufacturing. Int J Prod Econ. https://doi.org/10.1016/j.ijpe.2018.11.007

Li Y, Jia G, Cheng Y, Hu Y (2017) Additive manufacturing technology in spare parts supply chain: a comparative study. Int J Prod Res 55(5):1498-1515. https://doi.org/10.1080/00207543.2016.12314 33

Liu P, Huang SH, Mokasdar A, Zhou H, Hou L (2014) The impact of additive manufacturing in the aircraft spare parts supply chain: supply chain operation reference (scor) model based analysis. Prod Plan Control 25(13-14):1169-1181. https://doi.org/10.1080/09537287.2013.808835

McLearen LJ (2015) Additive manufacturing in the Marine Corps. Ph.D. thesis, Naval Postgraduate School, https://calhoun.nps.edu/handle/10945/45903

Mettas A (2000) Reliability allocation and optimization for complex systems. In: Annual reliability and maintainability symposium. 2000 Proceedings. International symposium on product quality and integrity (Cat. No.00CH37055). IEEE, pp 216-221, https://doi.org/10.1109/RAMS.2000.816310 
Minner S (2003) Multiple-supplier inventory models in supply chain management: a review. Int J Prod Econ 81-82:265-279. https://doi.org/10.1016/S0925-5273(02)00288-8

Moinzadeh K, Schmidt CP (1991) An (S-1, S) Inventory System with Emergency Orders. Oper Res 39(2):308-321. https://doi.org/10.1287/opre.39.2.308

Nicolai RP, Dekker R (2008) Optimal maintenance of multi-component systems: a review. In: Complex system maintenance handbook. Springer London, London, pp 263-286, https://doi.org/10.1007/9781-84800-011-7_11

Olde Keizer MC, Flapper SDP, Teunter RH (2017a) Condition-based maintenance policies for systems with multiple dependent components: A review. Eur J Oper Res 261(2):405-420. https://doi. org/10.1016/j.ejor.2017.02.044

Olde Keizer MC, Teunter RH, Veldman J (2017b) Joint condition-based maintenance and inventory optimization for systems with multiple components. Eur J Oper Res 257(1):209-222. https://doi. org/10.1016/j.ejor.2016.07.047

Öner K, Kiesmüller G, van Houtum GJ (2010) Optimization of component reliability in the design phase of capital goods. Eur J Oper Res 205(3):615-624. https://doi.org/10.1016/j.ejor.2010.01.030

Park KS (1981) (S - 1. IEEE Transactions on Reliability, S) Spare-Part Inventory Policy for Fleet Maintenance. https://doi.org/10.1109/TR.1981.5221182

Pérès F, Noyes D (2006) Envisioning e-logistics developments: making spare parts in situ and on demand. Comput Ind 57(6):490-503. https://doi.org/10.1016/j.compind.2006.02.010

Petchrompo S, Parlikad AK (2019) A review of asset management literature on multi-asset systems. Reliab Eng Syst Saf. https://doi.org/10.1016/j.ress.2018.09.009

Scheller-Wolf A, Veeraraghavan SK, van Houtum G-J (2006) Inventory policies with expedited ordering: single indexpolicies. Working paper, Wharton at the University of Pennsylvania, Philadelphia

Sherbrooke CC (2004) Optimal Inventory Modeling of Systems, International Series in Operations Research \& Management Science, vol 72. Springer, US, Boston, MA, https://doi.org/10.1007/b1098 56

Shouche S, Wysk RA, King RE, Harrysson OL (2016) Supply chain operations reference model for U.S. based powder bed metal additive manufacturing processes. In: 2016 winter simulation conference (WSC). IEEE, pp 1158-1169, https://doi.org/10.1109/WSC.2016.7822173

Sirichakwal I, Conner B (2016) Implications of additive manufacturing for spare parts inventory. 3D Print Addit Manuf 3(1):56-63. https://doi.org/10.1089/3dp.2015.0035

Sleptchenko A, Van der Heijden M (2016) Joint optimization of redundancy level and spare part inventories. Reliab Eng Syst Saf 153:64-74. https://doi.org/10.1016/j.ress.2016.04.006

Sleptchenko A, Johnson M (2015) Maintaining secure and reliable distributed control systems. INFORMS J Comput 27(1):103-117. https://doi.org/10.1287/ijoc.2014.0613

Song JS, Zhang Y (2016) Stock or print? Impact of 3D printing on spare parts logistics. https://doi. org/10.2139/ssrn.2884459

Song JS, Zipkin P (2009) Inventories with multiple supply sources and networks of queues with overflow bypasses. Manag Sci 55(3):362-372. https://doi.org/10.1287/mnsc.1080.0941

Song JS, Xiao L, Zhang H, Zipkin P (2017) Optimal Policies for a Dual-Sourcing Inventory Problem with Endogenous stochastic lead times. Oper Res 65(2):379-395. https://doi.org/10.1287/opre.2016.1557

Thomas DS, Gilbert SW (2014) Costs and cost effectiveness of additive manufacturing. Tech. Rep. 6, National Institute of Standards and Technology, Gaithersburg, MD, https://doi.org/10.6028/NIST. SP. 1176

Van Horenbeek A, Buré J, Cattrysse D, Pintelon L, Vansteenwegen P (2013) Joint maintenance and inventory optimization systems: a review. Int J Prod Econ. https://doi.org/10.1016/j.ijpe.2012.04.001

Van Houtum GJ, Kranenburg B (2015) Spare parts inventory control under system availability constraints, International Series in Operations Research \& Management Science, vol 227. Springer, US, Boston, MA, https://doi.org/10.1007/978-1-4899-7609-3

Veeraraghavan S, Scheller-Wolf A (2008) Now or later: a simple policy for effective dual sourcing in capacitated systems. Oper Res 56(4):850-864. https://doi.org/10.1287/opre.1080.0552

Walter M, Holmström J, Tuomi H, Yrjölä H (2004) Rapid manufacturing and its impact on supply chain management. In: Proceedings of the logistics research network annual conference

Wang H (2002) A survey of maintenance policies of deteriorating systems. Eur J Oper Res. https://doi. org/10.1016/S0377-2217(01)00197-7

Weller C, Kleer R, Piller FT (2015) Economic implications of 3D printing: market structure models in light of additive manufacturing revisited. Int J Prod Econ 164:43-56. https://doi.org/10.1016/j. ijpe.2015.02.020 
Westerweel B, Basten RJ, van Houtum GJ (2018) Traditional or additive manufacturing? Assessing component design options through lifecycle cost analysis. Eur J Oper Res 270(2):570-585. https://doi. org/10.1016/j.ejor.2018.04.015

Whittemore AS, Saunders SC (1977) Optimal inventory under stochastic demand with two supply options. SIAM J Appl Math 32(2):293-305. https://doi.org/10.1137/0132023

Wits WW, García JRR, Becker JMJ (2016) How additive manufacturing enables more sustainable enduser maintenance, repair and overhaul (MRO) strategies. Procedia CIRP 40:693-698. https://doi. org/10.1016/j.procir.2016.01.156

Zhou SX, Yang C (2016) Continuous-Review ( R, nQ ) Policies for Inventory Systems with Dual delivery modes. Oper Res 64(6):1302-1319. https://doi.org/10.1287/opre.2016.1538

Publisher's Note Springer Nature remains neutral with regard to jurisdictional claims in published maps and institutional affiliations. 